\title{
Neutralino dark matter in the left-right supersymmetric model
}

\author{
Durmuş A. Demir, ${ }^{1, *}$ Mariana Frank, ${ }^{2, \dagger}$ and Ismail Turan ${ }^{2, \sharp}$ \\ ${ }^{1}$ Department of Physics, Izmir Institute of Technology, IZTECH, TR35430, Izmir, Turkey \\ ${ }^{2}$ Department of Physics, Concordia University, 7141 Sherbrooke Street West, Montreal, Quebec, Canada H4B 1 R6
}

(Received 24 April 2006; published 8 June 2006)

\begin{abstract}
We study the neutralino sector of the left-right supersymmetric model. In addition to the possibilities available in the minimal supersymmetric model, the neutralino states can be superpartners of the $U(1)_{B-L}$ gauge boson, the neutral $S U(2)_{R}$ gauge boson, or of the Higgs triplets. We analyze neutralino masses and determine the parameter regions for which the lightest neutralino can be one of the new pure states. We then calculate the relic density of the dark matter for each of these states and impose the constraints coming from the $\rho$ parameter, the anomalous magnetic moment of the muon, $b \rightarrow s \gamma$, as well as general supersymmetric mass bounds. The lightest neutralino can be the bino, or the right-wino, or the neutral triplet Higgsino, all of which have different couplings to the standard model particles from the usual neutralinos. A light bino satisfies all the experimental constraints and would be the preferred dark matter candidate for light supersymmetric scalar masses, while the right-wino would be favored by intermediate supersymmetric mass scales. The neutral triplet Higgs fermion satisfies the experimental bounds only in a small region of the parameter space, for intermediate to heavy supersymmetric scalar masses.
\end{abstract}

DOI: 10.1103/PhysRevD.73.115001

PACS numbers: $12.60 . \mathrm{Cn}, 12.60 . \mathrm{Fr}, 14.80 . \mathrm{Ly}$

\section{INTRODUCTION}

Recent measurements from the Wilkinson Microwave Anisotropy Probe (WMAP) satellite [1] have shown that the cold dark matter (CDM) abundance in the universe is $\Omega_{\mathrm{CDM}} h^{2}=0.1126_{-0.00904}^{+0.00805}$, where $\Omega_{\mathrm{CDM}}$ is the density of the CDM species versus the critical density, and $h$ is the present value of the Hubble parameter. This corresponds to about $22 \%$ of the energy density of the universe being present in the form of dark matter.

The question of its nature and composition has been an open problem for some time. A candidate for dark matter must be stable, or long-living; it must be electrically and color neutral, as required by astrophysical constraints; and it must be nonrelativistic, which means that it should be massive. Since the relic density can be connected to the (thermally averaged) annihilation cross section of a dark matter candidate, its value indicates that these particles are interacting weakly. That is, dark matter candidates must be weakly interacting massive particles. Scenarios beyond the standard model provide several such exotic particles. Supersymmetry, in particular, provides the lightest neutralino as the lightest supersymmetric particle (LSP), which must be stable if $R$-parity is conserved. ${ }^{1}$

The minimal supersymmetric standard model (MSSM) Lagrangian, based on the symmetry group $S U(3)_{C} \otimes$ $S U(2)_{L} \otimes U(1)_{Y}$ is invariant under the $R$-parity discrete symmetry, under which all standard model particles are even, and their superpartners are odd. This parity is an essential element of the MSSM, and forbids baryon and

\footnotetext{
*Electronic address: demir@physics.iztech.edu.tr

${ }^{\dagger}$ Electronic address: mfrank@vax2.concordia.ca

\#Electronic address: ituran@physics.concordia.ca

${ }^{1}$ Gravitinos and axinos have also been studied as dark matter candidates, but we shall not consider this possibility here.
}

lepton number violating renormalizable couplings in the superpotential, which would lead to fast proton decay. Thus the $R$-parity is natural, and so is the possibility of having an LSP which emerges as a natural candidate for dark matter. In most supersymmetric scenarios, the LSP is the lightest neutralino [2].

Unfortunately there are problems with this CDM assignment in MSSM [3]. If one calculates the relic abundance of the lightest neutralino assumed to be a pure bino (the superpartner of the gauge boson of the $U(1)_{Y}$ gauge group), the relic density is too large (see [4] and references therein for a detailed discussion). To avoid this problem, one considers that there are several other supersymmetric particles of masses close to the LSP. If such sparticles exist, their coannihilation with the LSP leads to a reduction of the LSP relic abundance [5]. Extensive studies of such phenomena exist, and several publicly available codes such as DARKSUSY [6] and MICROMEGA [7], which include all the relevant coannihilation channels, are available for calculations within the MSSM. Cosmological and phenomenological constraints (such as $b \rightarrow s \gamma$ [8], $(g-2)_{\mu}$ [9], and Higgs mass bounds [10]) are also included. We could of course also abandon the pure bino state, and thus the constrained MSSM (CMSSM) which predicts it, and explore other versions of the lightest neutralino. However, this does not solve the problem because if one allows the lightest neutralino to be mostly left-wino, or Higgsino, the relic density becomes too small. Ways to avoid this exist as well, such as considering nonuniversal gaugino masses, abandoning the pure states and examining mixed states (such as bino-Higgsino), or more annihilations [11].

An alternative option is to explore scenarios beyond the MSSM. The extensions of the MSSM, motivated by the need to a dynamical solution to the naturalness problem associated with the $\mu$ parameter, offer novel CDM candidates not necessarily belonging to gaugino or Higgsino 
sectors of the MSSM. In next-to-minimal model (NMSSM) [12], for instance, the neutralino can be extremely light because it is dominated by its singlino component, which does not couple to SM particles (except for Higgs doublets). The existence of a very light $C P$-odd Higgs boson provides the possibility for this neutralino to annihilate sufficiently to avoid being overproduced in the early universe. Similar features are also found in $U(1)^{\prime}$ models [13] or in more general extensions [14]. This raises the hope that models beyond MSSM, preferably comprising the neutrino masses as well, can provide viable scenarios for alternative neutralinos which have different features than those in MSSM.

We propose here to look at the left-right supersymmetric model (LRSUSY) for candidates for dark matter. The advantages of the LRSUSY are many, such as combining supersymmetry with right-handed symmetry, and thus providing a mechanism for neutrino masses with the seesaw within a supersymmetric scenario. From the point of view of dark matter, the LRSUSY model is interesting because, unlike in MSSM, the usual explicit $R$-parity violating terms are forbidden in the superpotential by the symmetries of the model [15]. ${ }^{2}$ Thus the model naturally predicts a lightest supersymmetrical particle.

The LRSUSY model emerges naturally in some superstring theories [17], or in the breaking of such SUSY GUT scenarios, such as $S O(10)$ [18]. LRSUSY is based on the symmetry group $S U(3) \otimes S U(2)_{L} \otimes S U(2)_{R} \otimes U(1)_{V=B-L}$ and thus provides several new sources for exotic neutralinos, in the (neutral) partners of the $S U(2)_{R}$ or $U(1)_{B-L}$ gauge bosons, as well as the fermionic partners of several Higgs bosons. We analyze the candidates for dark matter in this model to see if they can avoid the problems that plague MSSM. After briefly reviewing the model in Sec. II, we perform a comprehensive study of neutralino mass eigenstates in Sec. III, followed by a calculation of relic density in Sec. IV. We look at the simplest scenario, that of pure states and avoid coannihilation with other supersymmetric particles by choosing the supersymmetric masses accordingly. We include constraints from the WMAP, $b \rightarrow s \gamma$, $\Delta \rho$, and muon $g-2$ as well as from experimental bounds on supersymmetric masses from direct collider searches.

\section{THE LRSUSY MODEL}

The most general superpotential for the group $S U(3) \otimes$ $S U(2)_{L} \otimes S U(2)_{R} \otimes U(1)_{V=B-L}$ is [19]:

$$
\begin{aligned}
W= & \mathbf{Y}_{Q}^{(i)} Q^{T} \Phi_{i} i \tau_{2} Q^{c}+\mathbf{Y}_{L}^{(i)} L^{T} \Phi_{i} i \tau_{2} L^{c}+i\left(\mathbf{Y}_{L R} L^{T} \tau_{2} \delta_{L} L\right. \\
& \left.+\mathbf{Y}_{L R} L^{c T} \tau_{2} \Delta_{R} L^{c}\right)+\mu_{L R}\left[\operatorname{Tr}\left(\Delta_{L} \delta_{L}+\Delta_{R} \delta_{R}\right)\right] \\
& +\mu_{i j} \operatorname{Tr}\left(i \tau_{2} \Phi_{i}^{T} i \tau_{2} \Phi_{j}\right)+W_{N R},
\end{aligned}
$$

where $W_{N R}$ denotes (possible) nonrenormalizable terms

\footnotetext{
${ }^{2}$ Note however that the $R$-parity can be broken spontaneously [16].
}

arising from higher scale physics or Planck scale effects $[20,21]$. Here the matter fields are defined as

$$
\begin{aligned}
& Q=\left(\begin{array}{l}
u \\
d
\end{array}\right) \sim(3,2,1,1 / 3), \quad Q^{c}=\left(\begin{array}{l}
d^{c} \\
u^{c}
\end{array}\right) \sim\left(3^{*}, 1,2,-1 / 3\right), \\
& L=\left(\begin{array}{l}
\nu \\
e
\end{array}\right) \sim(1,2,1,-1), \quad L^{c}=\left(\begin{array}{l}
e^{c} \\
\nu^{c}
\end{array}\right) \sim(1,1,2,1),
\end{aligned}
$$

where the numbers in the brackets denote the quantum numbers under $S U(3)_{C} \otimes S U(2)_{L} \otimes S U(2)_{R} \otimes U(1)_{B-L}$. The Higgs sector consists of the bidoublet and triplet Higgs superfields:

$$
\begin{aligned}
& \Phi_{1}=\left(\begin{array}{ll}
\phi_{11}^{0} & \phi_{11}^{+} \\
\phi_{12}^{-} & \phi_{12}^{0}
\end{array}\right) \sim(1,2,2,0), \\
& \Phi_{2}=\left(\begin{array}{ll}
\phi_{21}^{0} & \phi_{21}^{+} \\
\phi_{22}^{-} & \phi_{22}^{0}
\end{array}\right) \sim(1,2,2,0), \\
& \Delta_{L}=\left(\begin{array}{cc}
\frac{\Delta_{L}^{-}}{\sqrt{2}} & \Delta_{L}^{0} \\
\Delta_{L}^{--} & -\frac{\Delta_{L}^{-}}{\sqrt{2}}
\end{array}\right) \sim(1,3,1,-2), \\
& \delta_{L}=\left(\begin{array}{ll}
\frac{\delta_{L}^{+}}{\sqrt{2}} & \delta_{L}^{++} \\
\delta_{L}^{0} & -\frac{\delta_{L}^{+}}{\sqrt{2}}
\end{array}\right) \sim(1,3,1,2), \\
& \Delta_{R}=\left(\begin{array}{cc}
\frac{\Delta_{R}^{-}}{\sqrt{2}} & \Delta_{R}^{0} \\
\Delta_{R}^{--} & -\frac{\Delta_{R}^{-}}{\sqrt{2}}
\end{array}\right) \sim(1,1,3,-2), \\
& \delta_{R}=\left(\begin{array}{cc}
\frac{\delta_{R}^{+}}{\sqrt{2}} & \delta_{R}^{++} \\
\delta_{R}^{0} & -\frac{\delta_{R}^{+}}{\sqrt{2}}
\end{array}\right) \sim(1,1,3,2) .
\end{aligned}
$$

where $\Delta_{L}$ and $\delta_{R}$ are introduced in the model to cancel the fermionic anomalies introduced by the fermionic partners of $\delta_{L}$ and $\Delta_{R}$. The vev's of the Higgs fields in the LRSUSY can be chosen:

$$
\begin{array}{ll}
\left\langle\Phi_{1}\right\rangle=\left(\begin{array}{cc}
\kappa_{1} & 0 \\
0 & 0
\end{array}\right), & \left\langle\Phi_{2}\right\rangle=\left(\begin{array}{cc}
0 & 0 \\
0 & \kappa_{2}
\end{array}\right), \\
\left\langle\Delta_{L}\right\rangle=\left(\begin{array}{cc}
0 & v_{\Delta_{L}} \\
0 & 0
\end{array}\right), & \left\langle\delta_{L}\right\rangle=\left(\begin{array}{cc}
0 & 0 \\
v_{\delta_{L}} & 0
\end{array}\right), \\
\left\langle\Delta_{R}\right\rangle=\left(\begin{array}{cc}
0 & v_{\Delta_{R}} \\
0 & 0
\end{array}\right), & \left\langle\delta_{R}\right\rangle=\left(\begin{array}{cc}
0 & 0 \\
v_{\delta_{R}} & 0
\end{array}\right) .
\end{array}
$$

Some comments and explanations about the vev's chosen are required: $\kappa_{1}$ and $\kappa_{2}$ are the vev's of the MSSM-like Higgs bosons. They are responsible for giving masses to the quarks and leptons and they also contribute to $M_{W_{L}}$. We take the vev's of $\phi_{12}^{0}$ and $\phi_{21}^{0}$ to be zero because they induce FCNC at tree level (in both the leptonic and hadronic systems), as well as being responsible for $W_{L}-W_{R}$ mixing. The vev's could also have a phase which induces $C P$ violation, which is severely restricted in the kaon system. The non-MSSM Higgs vev's, $v_{\delta_{L}}$ and $v_{\Delta_{R}}$ are responsible for neutrino masses. For one generation (see [22], also $[20,23,24])$ 


$$
m_{\nu}=Y_{L R} v_{\delta_{L}}-\frac{\left(Y_{L}^{(\nu)}\right)^{2} \kappa_{1}^{2}}{Y_{L R} v_{\Delta_{R}}},
$$

where $v_{\delta_{L}}$ must be very small and $v_{\Delta_{R}}$ large, phenomenologically. In addition, $v_{\Delta_{L}}$ and $v_{\delta_{L}}$ enter in the formula for the mass of $W_{L}$ (or the $\rho$ parameter), while $v_{\Delta_{R}}, v_{\delta_{R}}$ enter in the formula for the mass of $W_{R}$. It is thus justified to take $v_{\Delta_{L}}, v_{\delta_{L}}$ to be negligibly small. For $v_{\Delta_{R}}$ there are two possibilities: either $v_{\Delta_{R}}$ is $\approx 10^{13} \mathrm{GeV}$ [24,25], which supports the seesaw mechanism, leptogenesis and provides masses for the light neutrinos in agreement with experimental constraints, but offers no hope to see right-handed particles; or $v_{\Delta_{R}}$ is $\approx 1-10 \mathrm{TeV}$, but one must introduce something else (generally an intermediate scale, or an extra symmetry) to make the neutrinos light $[15,20,25]$. Note that both $v_{\Delta_{R}}$ and $v_{\delta_{R}}$ contribute to the mass of $W_{R}$ [26], but only one needs to be heavy. Since $v_{\Delta_{R}}$ is responsible for heavy right-handed neutrino masses, it must be large. Thus $v_{\delta_{R}}$ is not constrained by the data. The mass term for neutralinos is given by

$$
\mathcal{L}_{N}=-\frac{1}{2} \psi^{0 T} Y \psi^{0}+\text { H.c. },
$$

where $\psi^{0}=$ $\left(-i \lambda_{V},-i \lambda_{L}^{0}, \tilde{\phi}_{11}^{0}, \tilde{\phi}_{22}^{0}, \tilde{\Delta}_{R}^{0}, \tilde{\delta}_{R}^{0},-i \lambda_{R}^{0}, \tilde{\phi}_{12}^{0}, \tilde{\phi}_{21}^{0}\right)^{T}$. Here $\lambda_{V}$ is the $U(1)_{B-L}$ bino, $\lambda_{L}^{0}$ the left-handed neutral $\left[S U(2)_{L}\right]$ wino, and $\lambda_{R}^{0}$ the right-handed neutral $\left[S U(2)_{R}\right]$ wino. The rest of the fields are Higgsinos. The neutralino mass matrix $Y$ is equal to [27]

$$
Y=\left(\begin{array}{c|cccc|ccccc} 
& -i \lambda_{V} & -i \lambda_{L}^{0} & \tilde{\phi}_{11}^{0} & \tilde{\phi}_{22}^{0} & \tilde{\Delta}_{R}^{0} & \tilde{\delta}_{R}^{0} & -i \lambda_{R}^{0} & \tilde{\phi}_{12}^{0} & \tilde{\phi}_{21}^{0} \\
\hline-i \lambda_{V} & M_{V} & 0 & 0 & 0 & -\sqrt{2} g_{V} v_{\Delta} & \sqrt{2} g_{V} v_{\delta} & 0 & 0 & 0 \\
-i \lambda_{L}^{0} & 0 & M_{L} & \frac{g_{L} \kappa_{1}}{\sqrt{2}} & -\frac{g_{L} \kappa_{2}}{\sqrt{2}} & 0 & 0 & 0 & 0 & 0 \\
\tilde{\phi}_{11}^{0} & 0 & \frac{g_{L} \kappa_{1}}{\sqrt{2}} & 0 & -\mu_{1} & 0 & 0 & -\frac{g_{R} \kappa_{1}}{\sqrt{2}} & 0 & 0 \\
\tilde{\phi}_{22}^{0} & 0 & -\frac{g_{L} \kappa_{2}}{\sqrt{2}} & -\mu_{1} & 0 & 0 & 0 & \frac{g_{R} \kappa_{2}}{\sqrt{2}} & 0 & 0 \\
\hline \tilde{\Delta}_{R}^{0} & -\sqrt{2} g_{V} v_{\Delta} & 0 & 0 & 0 & 0 & \mu_{2} & -\sqrt{2} g_{R} v_{\Delta} & 0 & 0 \\
\tilde{\delta}_{R}^{0} & \sqrt{2} g_{V} v_{\delta} & 0 & 0 & 0 & \mu_{2} & 0 & -\sqrt{2} g_{R} v_{\delta} & 0 & 0 \\
-i \lambda_{R}^{0} & 0 & 0 & -\frac{g_{R} \kappa_{1}}{\sqrt{2}} & \frac{g_{R} \kappa_{2}}{\sqrt{2}} & \sqrt{2} g_{R} v_{\Delta} & -\sqrt{2} g_{R} v_{\delta} & \mathrm{M}_{R} & 0 & 0 \\
\tilde{\phi}_{12}^{0} & 0 & 0 & 0 & 0 & 0 & 0 & 0 & 0 & -\mu_{1} \\
\tilde{\phi}_{21}^{0} & 0 & 0 & 0 & 0 & 0 & 0 & 0 & -\mu_{1} & 0
\end{array}\right)
$$

where $\mu_{i j}=\mu_{1},(i \neq j)$ and $\mu_{L R} \equiv \mu_{2}$ are assumed. ${ }^{3}$ The upper $4 \times 4$ part of the matrix contains MSSM-like states. There are still too many unknown parameters and for further simplification one can define the following:

$$
\begin{aligned}
& g_{L}=g_{R} \equiv g, \quad g_{V}=\frac{e}{\sqrt{\cos 2 \theta_{W}}}, \\
& \sqrt{\kappa_{1}^{2}+\kappa_{2}^{2}}=174 \mathrm{GeV}, \quad \tan \beta=\frac{\kappa_{2}}{\kappa_{1}} .
\end{aligned}
$$

If we assume that GUT relations between gaugino masses hold, we can simplify the parameters further, but for now we keep them general and discuss specific scenarios later.

Two roots of the characteristic equation for $Y$ are already known, $\pm \mu_{1}$, and we are left with a 7 th order equation to solve. There is no exact solution and a numerical or semiexact approach is necessary. Whereas there are constraints on $M_{W_{R}}$ and $M_{Z_{R}}$ [28], there are no constraints on $M_{V}$, the

\footnotetext{
${ }^{3}$ From now on, we drop the subscript " $R$ " from $v_{\Delta_{R}}, v_{\delta_{R}}$.
}

$U(1)_{B-L}$ gaugino mass, or on $M_{L(R)}$, the $S U(2)_{L(R)}$ gaugino mass parameter.

Basically the number of free parameters can be chosen as $M_{V}, M_{L}, M_{R}, v_{\Delta}, v_{\delta}, \mu_{1}, \mu_{2}$, and $\tan \beta$. So, we could look at under what circumstances the lightest neutralino becomes a mainly pure state (bino, left-wino, right-wino, or Higgsino) or a mixed state as bino-Higgsino and rightwino-Higgsino.

\section{THE CLASSIFICATION OF SCENARIOS}

Starting from the neutralino mass matrix, we can classify the following cases: ${ }^{4}$

(1) The lightest neutralino is mostly bino $\left(\lambda_{V}\right.$ in our notation). Note that this is the $B-L$ gaugino, not to be confused with the $U(1)_{Y}$ MSSM bino. To obtain

\footnotetext{
${ }^{4} \mathrm{We}$ set $v_{\Delta}=1.5 \mathrm{TeV}$ for all the cases unless otherwise stated. Note also that there will be no rigorous model constraint analysis on the masses of the LSP in this section. We leave that to the next section.
} 
this bino as the lightest state, we must have $M_{V} \approx$ $50-100 \mathrm{GeV}$ and $v_{\delta} \approx 50-100 \mathrm{GeV}$ (both light) while $\mu_{2} \approx 1 \mathrm{TeV}$ or larger (heavy). Decreasing $\mu_{2}$ to $200-500 \mathrm{GeV}$, the lightest state becomes a mixture of $\lambda_{V}$ and $\tilde{\delta}_{R}^{0}$.

(2) The lightest neutralino is mostly a right-handed wino $\left(\lambda_{R}^{0}\right.$ in our notation). To obtain this, the masses of the other two gauginos must be larger than that of $\lambda_{R}^{0}: M_{V}, M_{L} \approx 600 \mathrm{TeV}$ and $v_{\delta} \approx 50-100 \mathrm{GeV}$ is light; while $\mu_{1} \approx 1 \mathrm{TeV}, \quad \mu_{2} \approx 3-5 \mathrm{TeV}$. Decreasing $\mu_{2}$ to $1 \mathrm{TeV}$, the lightest state becomes a mixture of $\lambda_{R}^{0}, \lambda_{V}$, and $\tilde{\delta}_{R}^{0}$.

(3) The lightest neutralino is the right-handed Higgsino $\tilde{\delta}_{R}^{0}$. This scenario is obtained for a large range of parameters, as long as both $\mu_{2}$ and $v_{\delta}$ are small, $\approx$ $200 \mathrm{GeV}$ or larger, and $(0,100) \mathrm{GeV}$, respectively. In fact, this requirement is satisfied for a wide portion of the parameter space, as long as $\mu_{2}$ and $v_{\delta}$ are smaller than other parameters in the theory. This scenario is interesting since $\tilde{\delta}_{R}^{0}$ does not couple to any standard model particles (or their SUSY partners). As explained previously, $v_{\delta}$ is not constrained by the data.

(4) The lightest neutralino could be a mixture of $\lambda_{V}, \tilde{\delta}_{R}^{0}$, and $\tilde{\Delta}_{R}^{0}$. In this case we can get eigenvectors and eigenvalues analytically and calculate the relic density for the mixed state. This is a generalization of the first case, where both $\tilde{\Delta}_{R}^{0}$ and $\tilde{\delta}_{R}^{0}$ are included. Here we can take advantageous ratios of the vevs of $\tilde{\delta}_{R}^{0}$ and $\tilde{\Delta}_{R}^{0}$ to get only one combination of $\tilde{\delta}_{R}^{0}$ and $\tilde{\Delta}_{R}^{0}$ mixed with the bino. This is the case if we assume the vev's $v_{\delta}$ and $v_{\Delta}$ equal.

(5) The lightest neutralino could be a mixture of $\lambda_{R}^{0}$ (the right-handed wino), and the non-MSSM Higgsinos $\tilde{\delta}_{R}^{0}, \tilde{\Delta}_{R}^{0}$. As opposed to the $B-L$ bino, the righthanded wino mixes with MSSM Higgsinos as well, which will be considered in a separate scenario. Again like in scenario (4), one can make the rightwino couple with one combination of $\tilde{\delta}_{R}^{0}$ and $\tilde{\Delta}_{R}^{0}$ if their vev's are assumed to be equal.

(6) Finally, the lightest neutralino could be a mixture of $\lambda_{R}^{0}$ with only the MSSM Higgsinos, $\tilde{\phi}_{11}^{0}$ and $\tilde{\phi}_{22}^{0}$. This can be the case if both $M_{R}$ and $\mu_{1}$ are small compared to $M_{L}, M_{V}, \mu_{2}$, and the vev's $v_{\Delta}$ and $v_{\delta}$. One could consider the $v_{\Delta}=v_{\delta}$ case and decouple $\tilde{\delta}_{R}^{0}$ and $\tilde{\Delta}_{R}^{0}$ by taking $\mu_{2}$ to be large.

We are not interested in the MSSM lightest neutralino scenarios (in which the left-wino or MSSM Higgsino mixed with left-wino are the LSP), since these have already been studied. As far as we can tell, scenarios 1-6 are the important (most striking) possibilities.

\section{A. The lightest neutralino state}

The following four sets of figures further illustrate the scenarios mentioned above. We assume the composition of the lightest state as (for the first three scenarios)

$$
\begin{aligned}
\left|\tilde{\chi}_{1}^{0}\right\rangle= & N_{11}\left|\lambda_{L}^{0}\right\rangle+N_{12}\left|\lambda_{R}^{0}\right\rangle+N_{13}\left|\lambda_{V}\right\rangle+N_{14}\left|\tilde{\phi}_{11}^{0}\right\rangle \\
& +N_{15}\left|\tilde{\phi}_{22}^{0}\right\rangle+N_{16}\left|\tilde{\Delta}_{R}^{0}\right\rangle+N_{17}\left|\tilde{\delta}_{R}^{0}\right\rangle .
\end{aligned}
$$

First we look at the possibilities for scenarios (1) and (3). Here we want the bino to be the lightest, so we take $M_{L}$, $M_{R}$, and $\mu_{1}$ large. Accordingly, we need $v_{\delta}$ to be small. Varying $\mu_{2}$ will take us from a mostly bino lightest state to a mostly $\tilde{\delta}_{R}^{0}$ lightest state.

In the left panel of Fig. 1, the difference between the bino and Higgsino compositions of the lightest neutralino state is shown as a function of $\mu_{2}$ by choosing $\left(M_{V}, v_{\delta}\right)=$ $(0,0),(0,100),(0,400)$, and $(200,400) \mathrm{GeV}$. The rest of the parameters are fixed as $M_{L}=M_{R}=600 \mathrm{GeV}, \mu_{1}=$ $500 \mathrm{GeV}, v_{\Delta}=1.5 \mathrm{TeV}$, and $\tan \beta=2$. In the right panel, the mass of the lightest neutralino is given as a function of $\mu_{2}$ for the same parameter values. As seen from the figure the lightest state is pure Higgsino $\tilde{\delta}_{R}^{0}$ for very small $\mu_{2}$ values, regardless of the values of $\left(M_{V}, v_{\delta}\right)$, as long as they are smaller than $500 \mathrm{GeV}$. The ratio is more sensitive to the vev of the right-Higgsino $\left(v_{\delta}\right)$ than to the $U(1)_{B-L}$ gaugino parameter $M_{V}$. At large $\mu_{2}$ the state becomes pure bino from pure Higgsino and its mass shows a strong dependence on $\mu_{2}$. So, one can consider these two limiting cases as realizations of the scenarios (1) and (3), mentioned above. From the right panel, one can conclude that, except for very small values of $\mu_{2}$, the cases where $M_{V}$ and $v_{\delta}$ are larger than $200 \mathrm{GeV}$ predict a lightest neutralino with a mass in the range of $200-300 \mathrm{GeV}$. Otherwise its mass remains less than $150 \mathrm{GeV}$.

Next we look at scenarios (2) and (3). Here we want the right-wino to be the lightest neutralino, so we take $M_{L}, M_{V}$, and $\mu_{1}$ large, and $v_{\delta}$ to be small. Varying $\mu_{2}$ will take us from a mostly right-wino lightest state to a mostly $\tilde{\delta}_{R}^{0}$ lightest state.

In Fig. 2, we plot the difference between the bino and the Higgsino compositions of the lightest neutralino state as a function of $\mu_{2}$ for various $\left(M_{R}, v_{\delta}\right)$ values, for $M_{L}=$ $M_{V}=600 \mathrm{GeV}, \mu_{1}=500 \mathrm{GeV}, v_{\Delta}=1.5 \mathrm{TeV}$, and $\tan \beta=2$. On the right panel, the mass of the lightest neutralino is shown as a function of $\mu_{2}$ for the same parameter values. The third diagram shows the bino composition of the lightest state. This case exhibits a very similar dependence on $\mu_{2}$ as the bino-Higgsino case. The curves for various $\left(M_{R}, v_{\delta}\right)$ pairs indicate that the ratio is insensitive to their chosen values, as long as they are assumed to be less than $200 \mathrm{GeV}$. The bino composition becomes significant only around $\mu_{2}=1 \mathrm{TeV}$, where the right-wino and Higgsino mix almost equally, and it is negligible as $\mu_{2}$ becomes larger. The contribution of the bino with respect to that of the right-wino is significant for very small $\mu_{2}$ values $(\sim 0 \mathrm{GeV})$ but such small values are excluded from the lower bound on the mass of the lightest chargino, which is around $90 \mathrm{TeV}$. The mass of the lightest state is less than $150 \mathrm{GeV}$ for $v_{\delta} \leq 200 \mathrm{GeV}$ if $M_{R}$ is very 

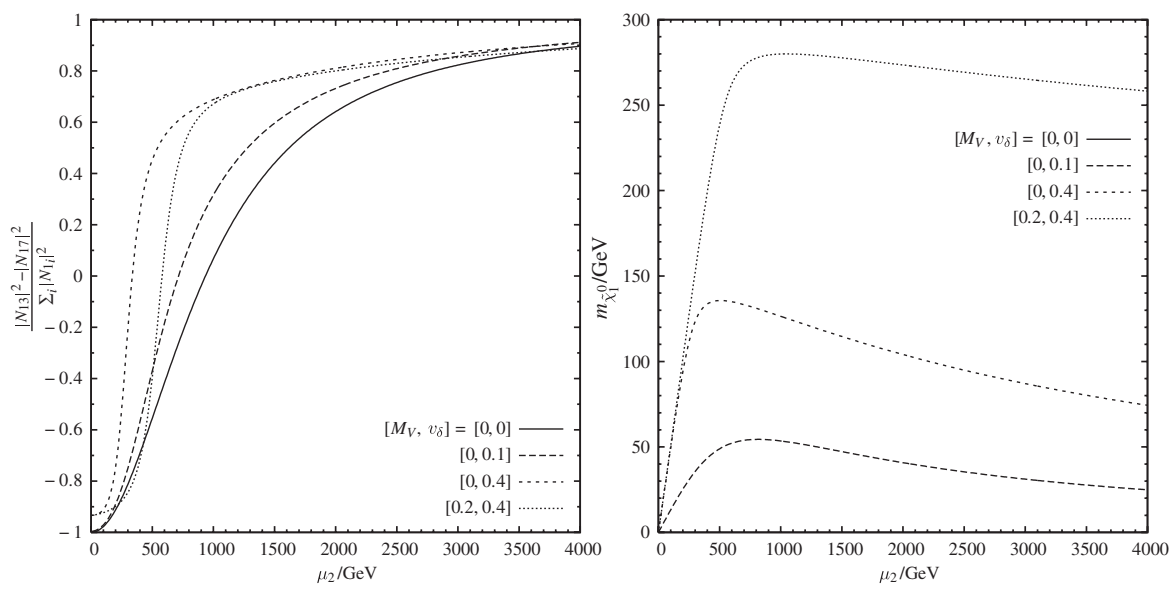

FIG. 1. On the left panel, the difference between bino and Higgsino compositions of the lightest neutralino state as a function of $\mu_{2}$ for various $\left(M_{V}, v_{\delta}\right)$ values in $\mathrm{TeV}$, for $M_{L}=M_{R}=600 \mathrm{GeV}, \mu_{1}=500 \mathrm{GeV}, v_{\Delta}=1.5 \mathrm{TeV}$, and $\tan \beta=2$. On the right panel, the mass of the lightest neutralino as a function of $\mu_{2}$ for the same parameter values.

small, or for $\left(M_{R}, v_{\delta}\right) \leq(50,50) \mathrm{GeV}$. Otherwise, it is larger than $200 \mathrm{GeV}$ for $\mu_{2}$ larger than $500 \mathrm{GeV}$. While the composition of the state is insensitive to $M_{R}$ and $v_{\delta}$, the mass of the lightest neutralino is sensitive to both. Here one can recover scenarios (2) and (3) in the limiting cases and the mixed state $\left(\left|\lambda_{R}^{0}\right\rangle+\left|\tilde{\delta}_{R}^{0}\right\rangle\right)$ in between.
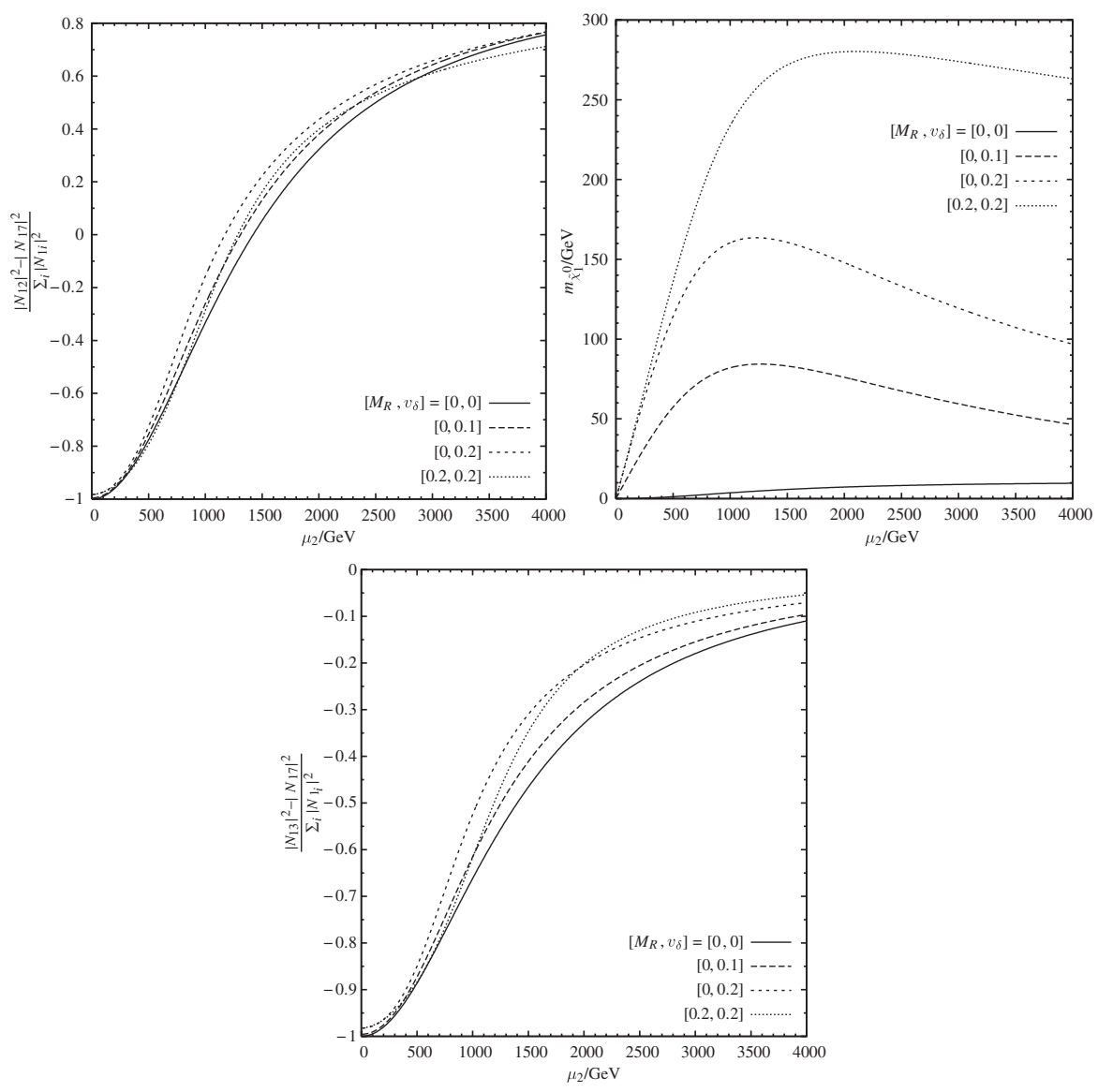

FIG. 2. On the left panel, the difference between right-wino and Higgsino compositions of the lightest neutralino state as a function of $\mu_{2}$ for various $\left(M_{R}, v_{\delta}\right)$ values in $\mathrm{TeV}$, for $M_{L}=M_{V}=600 \mathrm{GeV}, \mu_{1}=500 \mathrm{GeV}, v_{\Delta}=1.5 \mathrm{TeV}$, and tan $\beta=2$. On the right panel, the mass of the lightest neutralino as a function of $\mu_{2}$ for the same parameter values. The third diagram shows the difference between bino and Higgsino compositions of the lightest state. 
Finally, we look at realizations of scenarios (4), (5), and (6). Here we are interested in mixtures of bino-(nonMSSM) Higgsino, right-wino-(non-MSSM) Higgsino, and right-wino-MSSM Higgsino. We need to decouple the MSSM particles for scenarios (4) and (5), so we take $M_{L}$ and $\mu_{1}$ to be large. In addition we take $v_{\Delta}=v_{\delta}$ (as opposed to the previous scenarios, where we took one of them small). We vary $M_{V}, M_{R}$ and $\mu_{2}$ to go from one case to another.

The realization of the last three scenarios discussed above requires some radical changes in the parameter set and has a distinct structure. We first decouple the $\tilde{\phi}_{12}^{0}$ and $\tilde{\phi}_{21}^{0}$ fields. Then, without loss of generality, we rotate the basis

$$
\begin{aligned}
& \left\{\left|\lambda_{L}^{0}\right\rangle,\left|\lambda_{R}^{0}\right\rangle,\left|\lambda_{V}\right\rangle,\left|\tilde{\phi}_{11}^{0}\right\rangle,\left|\tilde{\phi}_{22}^{0}\right\rangle,\left|\tilde{\Delta}_{R}^{0}\right\rangle,\left|\tilde{\delta}_{R}^{0}\right\rangle\right\} \\
& \quad \rightarrow\left\{\left|\lambda_{L}^{0}\right\rangle,\left|\lambda_{R}^{0}\right\rangle,\left|\lambda_{V}\right\rangle,\left|\tilde{\phi}_{+}^{0}\right\rangle,\left|\tilde{\phi}_{-}^{0}\right\rangle,\left|\tilde{\eta}_{+}^{0}\right\rangle,\left|\tilde{\eta}_{-}^{0}\right\rangle\right\}
\end{aligned}
$$

such that

$$
\left|\tilde{\phi}_{ \pm}^{0}\right\rangle \equiv \frac{\left|\tilde{\phi}_{11}^{0}\right\rangle \pm\left|\tilde{\phi}_{22}^{0}\right\rangle}{\sqrt{2}}, \quad\left|\tilde{\eta}_{ \pm}^{0}\right\rangle \equiv \frac{\left|\tilde{\Delta}_{R}^{0}\right\rangle \pm\left|\tilde{\delta}_{R}^{0}\right\rangle}{\sqrt{2}}
$$

In this new basis the mass matrix $Y$ in Eq. (2.7) becomes

$$
Y^{\prime}=\left(\begin{array}{c|ccc|ccc} 
& -i \lambda_{L}^{0} & -i \lambda_{R}^{0} & -i \lambda_{V} & \tilde{\phi}_{+}^{0} & \tilde{\phi}_{-}^{0} & \tilde{\eta}_{-}^{0} \\
\hline-i \lambda_{L}^{0} & M_{L} & 0 & 0 & \frac{g_{L}}{2} \kappa^{-} & \frac{g_{L}}{2} \kappa^{-} & 0 \\
-i \lambda^{0} & 0 & M_{R} & 0 & -\frac{g_{R}}{2} \kappa^{+} & -\frac{g_{R}}{2} \kappa^{+} & 2 g_{R} v_{R} \\
-i \lambda_{V} & 0 & 0 & M_{V} & 0 & 0 & -2 g_{V} v_{R} \\
\hline \tilde{\phi}_{+}^{0} & \frac{g_{L}}{2} \kappa^{-} & -\frac{g_{R}}{2} \kappa^{-} & 0 & -\mu_{1} & 0 & 0 \\
\tilde{\phi}_{-}^{0} & \frac{g_{L}}{2} \kappa^{+} & -\frac{g_{R}}{2} \kappa^{+} & 0 & 0 & \mu_{1} & 0 \\
\tilde{\eta}_{-}^{0} & 0 & 2 g_{R} v_{R} & -2 g_{V} v_{R} & 0 & 0 & \mu_{2}
\end{array}\right),
$$

where we have assumed $v_{\Delta}=v_{\delta} \equiv v_{R}$, and further defined $\kappa^{ \pm}=\kappa_{1} \pm \kappa_{2}$. Under this assumption the rotated state $\tilde{\eta}_{+}^{0}$ decouples and only $\tilde{\eta}_{-}^{0}$ remains, which allows us to analyze scenarios (4), (5) and (6) more conveniently.

Figure 3 shows the difference between the right-wino (bino) and the rotated non-MSSM Higgsino ( $\tilde{\eta}_{-}^{0}$ ) compositions of the lightest neutralino state as functions of $\mu_{2}$ by choosing various $\left(M_{R}, M_{V}\right)$ values in the left (right) panel. The rest of the parameters are taken as $M_{L}=600 \mathrm{GeV}$, $\mu_{1}=5 \mathrm{TeV}, v_{\Delta}=v_{\delta} \equiv v_{R}=1 \mathrm{TeV}$, and $\tan \beta=2$. Here $\left|N_{16}^{-}\right|^{2}$ is the amount of the rotated Higgsino field $\tilde{\eta}_{-}^{0}$ in the lightest neutralino state. One can divide the discussion into two regions-one with small $M_{R} \leq$ $50 \mathrm{GeV}$ and $M_{V} \leq 1 \mathrm{GeV}$, and the other with larger $M_{R}$ and $M_{V}$. In the first part, neither the right-wino nor the bino is the lightest neutralino till $\mu_{2} \sim 500 \mathrm{GeV}$. The decoupled state $\tilde{\eta}_{+}^{0}$ is the lightest state (which cannot be seen from the figures ${ }^{5}$ ). For $\mu_{2}>500 \mathrm{GeV}$, the lightest state is mainly a right-wino or a right-wino-Higgsino mixture. Otherwise, for intermediate values of $\mu_{2}$, it is a mixture of right-wino, bino, and Higgsino $\tilde{\eta}_{-}^{0}$. For the second part where $M_{R}>50 \mathrm{GeV}$ and $M_{V}>1000 \mathrm{GeV}$, the curves are horizontal lines passing through zero for

\footnotetext{
${ }^{5}$ Note that the horizontal line passing through zero does not always mean equal mixing. One could obtain such a result for vanishing individual contributions as well, which is indeed the case here.
}

both graphs until $\mu_{2} \sim 2 \mathrm{TeV}$. In that region of the parameter space the lightest state is pure left-wino. The rightwino-Higgsino mixture is possible as the lightest neutralino state only after that point. The bino-Higgsino lightest state is not realized in this region. From the third diagram in Fig. 3, one can say that, within the range considered for $\mu_{2}$, the mass of the lightest state is greater than $300 \mathrm{GeV}$ for $\mu_{2} \geq 500 \mathrm{GeV}$. So, the scenarios (4) and (5) require the lightest state to be rather heavy unless $\mu_{2}$ is too large. This takes us to pure right-wino scenario. Including all the possible model constraints in the next section will restrict our parameter space further.

In Fig. 4, the difference between the composition of the lightest neutralino as a right-wino, or a MSSM Higgsino is shown on the left panel as a function of $M_{R}$ for various $\mu_{1}$ values in the range 0 to $500 \mathrm{GeV}$. The other parameters are chosen as $M_{L}=M_{V}=1 \mathrm{TeV}, \quad \mu_{2}=20 \mathrm{TeV}, \quad v_{\Delta}=$ $v_{\delta}=1 \mathrm{TeV}$, and $\tan \beta=2$. The ratio is sensitive to both $M_{R}$ and $\mu_{1}$. For values of $\mu_{1}$ smaller than $25 \mathrm{GeV}$, the lightest state is pure MSSM Higgsino and for values larger than $450 \mathrm{GeV}$ the state almost is pure right-wino, if $M_{R}$ is equal to, or less than, $150 \mathrm{GeV}$. Evenly mixed states are obtained for intermediate values of $\mu_{1}$. As $M_{R}$ becomes equal to, or larger than, $400 \mathrm{GeV}$, the lightest state remains pure right-wino for the most part of the $\mu_{1}$ range. As suggested by the right panel, the mass of the lightest state is very sensitive to the parameter $M_{R}$ and can be quite large for large $M_{R}$ and $\mu_{1}$ values. 

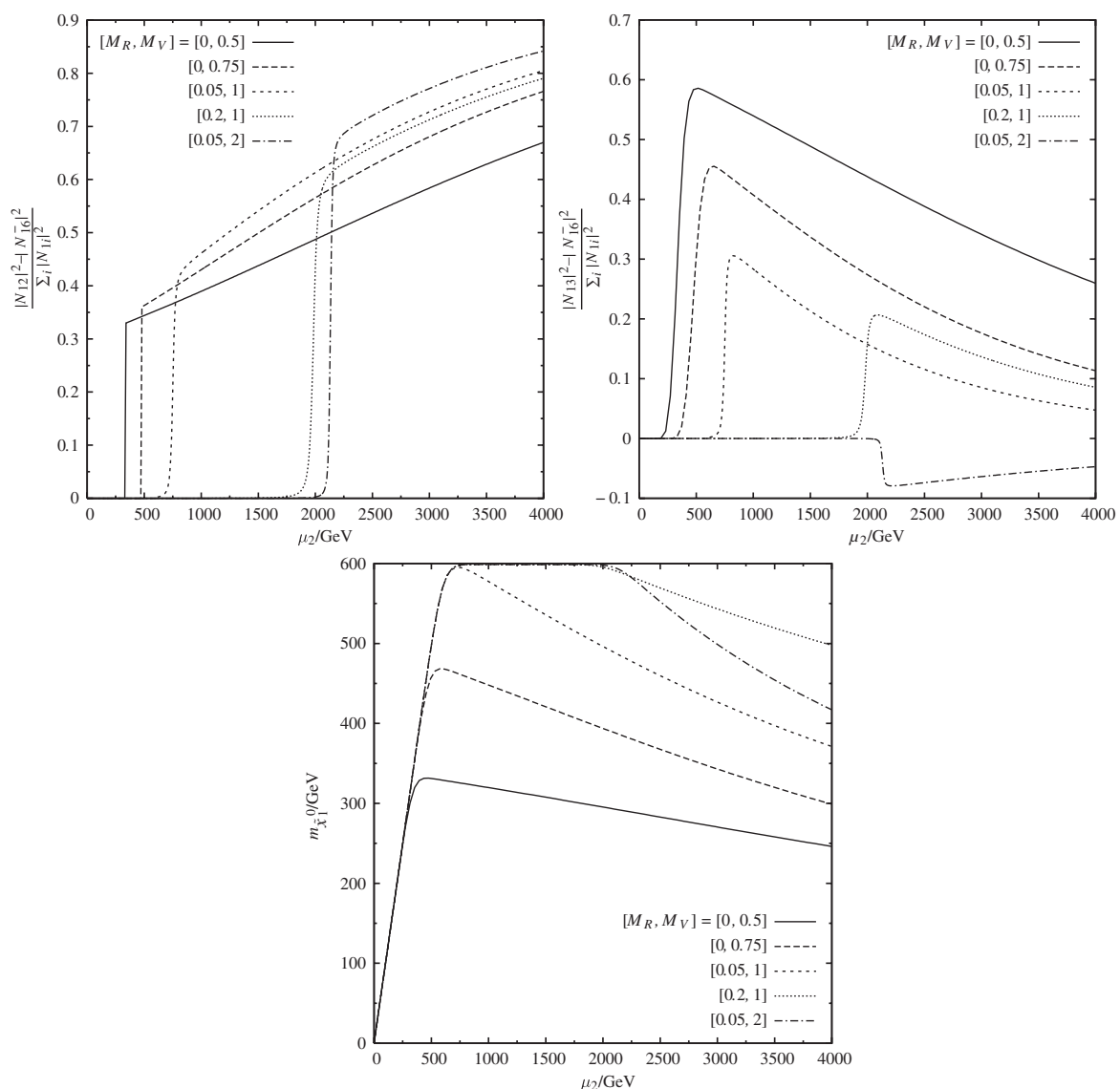

FIG. 3. On the left panel, the difference between right-wino and rotated non-MSSM-Higgsino compositions of the lightest neutralino state as a function of $\mu_{2}$ for various $\left(M_{R}, M_{V}\right)$ values in TeV, for $M_{L}=600 \mathrm{GeV}, \mu_{1}=5 \mathrm{TeV}, v_{\Delta}=v_{\delta}=1 \mathrm{TeV}$, and tan $\beta=2$. On the right panel, the difference between the bino and the rotated non-MSSM-Higgsino compositions as functions of $\mu_{2}$ for the same parameter values. The third diagram shows the mass of the lightest neutralino as a function of $\mu_{2}$.

This completes analyses of possible LSP candidates in LRSUSY for certain patterns of the model parameters. The figures illustrate the nature and purity of the lightest neutralino states as a function of various gaugino masses and
Higgs vevs. In the next section we will perform a detailed analysis of the relic abundance of LSP and its ability to explain the $\mathrm{CDM}$ in the universe.
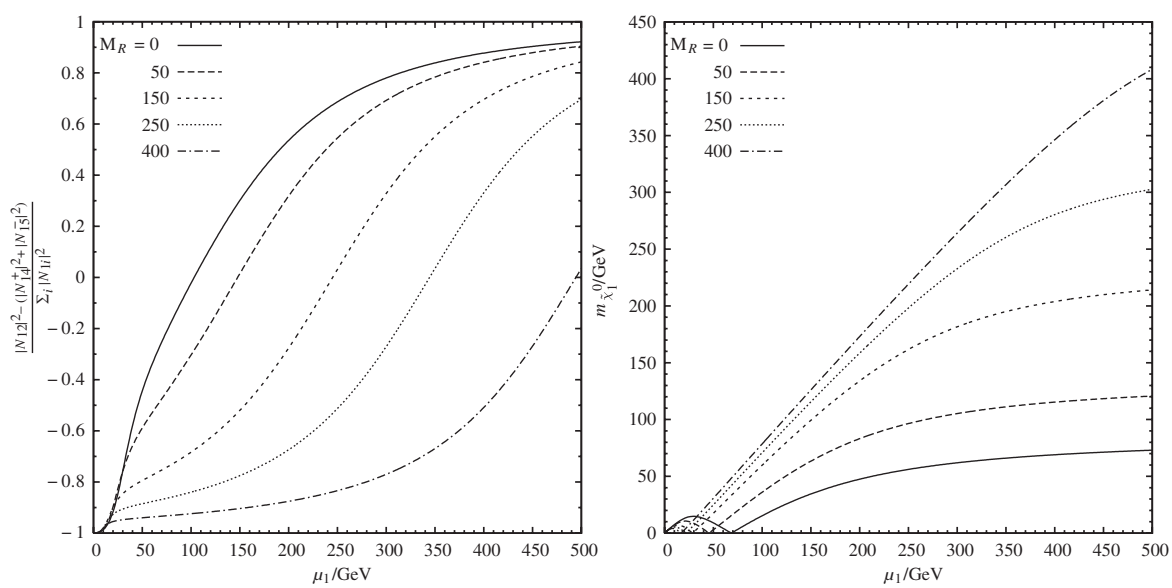

FIG. 4. On the left panel, the difference between right-wino and rotated MSSM-Higgsino compositions of the lightest neutralino state as functions of $\mu_{1}$ at various $M_{R}$ values in $\mathrm{GeV}$, for $M_{L}=M_{V}=1 \mathrm{TeV}, \mu_{2}=20 \mathrm{TeV}, v_{\Delta}=v_{\delta}=1 \mathrm{TeV}$, and tan $\beta=2$. On the right panel, the mass of the lightest neutralino as a function of $\mu_{1}$ for the same parameter values. 


\section{RELIC ABUNDANCE ANALYSIS OF CDM}

In this section we calculate the relic density of cold dark matter within the LRSUSY framework. Before presenting our results we give some details of the procedure followed.

The time evolution of the number density $n_{i}$ for a relic particle is given by the Boltzmann equation (taken within spatially flat Friedman-Robertson-Walker background). Furthermore, a single Boltzmann equation can be defined [5] for the total number density, $n \equiv \sum_{i} n_{i}$

$$
\dot{n}+3 h n=-\left\langle\sigma_{\text {eff }} v\right\rangle\left[n^{2}-n_{\text {eq }}^{2}\right],
$$

where $h$ is the Hubble parameter, $v$ is the relative velocity of the annihilating particles, and $n_{\text {eq }}$ is the number density corresponding to the sum of each species number density at thermal equilibrium (that is, the density in the early universe). Here $\sigma_{\text {eff }}$ is the properly averaged effective cross section of the CDM candidate into ordinary particles (i.e., SM particles including the Higgs bosons) and will be defined below. Clearly, " \langle\rangle " stands for the thermal average.

In this study we concentrate on regions of the parameter space where the coannihilation effects are not significant. The relativistic thermally averaged cross section times relative velocity reads as [29]

$$
\left\langle\sigma_{\mathrm{eff}} v\right\rangle(x)=\frac{\int_{4 m_{\chi}^{2}}^{\infty} d s \sigma(s) \sqrt{s}\left(s / 4-m_{\chi}^{2}\right) K_{1}\left(\sqrt{s} /\left(m_{\chi} x\right)\right)}{2 m_{\chi}^{5} x K_{2}(1 / x)^{2}},
$$

where $m_{\chi}$ is the mass of the annihilating particle, the lightest neutralino in our consideration; $K_{1}$ and $K_{2}$ are modified Bessel functions; $x \equiv T / m_{\chi}$ is the dimensionless temperature parameter; $\sqrt{s}$ is the center of mass energy of the annihilating neutralino pair; and $\sigma(s)$ is the cross section for the annihilation reaction $\chi_{1}^{0} \bar{\chi}_{1}^{0} \rightarrow X_{\mathrm{SM}}$, where $X_{\mathrm{SM}}$ represents all the allowed two-body SM final states. We will not approximate Eq. (4.2) by expanding $v$ in a Taylor series, as this approximation might not always be safe, especially for cases with fast varying integrands.

In general, there is no analytical solution to the Boltzmann equation, Eq. (4.1), which is a Riccati-type equation. Thus a numerical approach is required. There exist several different ways to proceed in the literature [7,29]. Here we define a freeze-out temperature parameter $x_{F} \equiv T_{F} / m_{\chi}$, which we use as an approximate solution to Eq. (4.1) [30] as

$$
n_{\chi}\left(T_{0}\right) \sim \frac{1}{m_{\chi} M_{\mathrm{Pl}}} \sqrt{\frac{4 \pi^{3} g *}{45}}\left(\frac{T_{\chi}}{T_{\gamma}}\right)^{3} T_{\gamma}^{3} \frac{1}{\int_{x_{0}}^{x_{F}} d x\left\langle\sigma_{\mathrm{eff}} v\right\rangle(x)},
$$

where $M_{\mathrm{Pl}}$ is the Planck mass, $T_{0}$ is today's temperature $(\sim 2.742 \mathrm{~K})$, so that $x_{0}$ can be approximated as zero; $T_{\chi}\left(T_{\gamma}\right)$ is the present temperature of the neutralino (cosmic microwave background), and $g *$ represents the SM effective number of degrees of freedom at the freeze-out temperature ( $g * \sim 81$ is used). Note that using an approximate solution of the Boltzmann equation instead of solving it numerically will introduce an uncertainty of up to $10 \%$ into our results.

The freeze-out temperature parameter $x_{F}$ can be obtained from the following transcendental equation:

$$
\frac{1}{x_{F}}=\log \left[\frac{m_{\chi} M_{\mathrm{Pl}}}{2 \pi^{3}} \sqrt{\frac{45}{2 g *}} \sqrt{x_{F}}\left\langle\sigma_{\mathrm{eff}} v\right\rangle\left(x_{F}\right)\right]
$$

which can be solved iteratively. As a starting point we need to choose a value for $x_{F}$. We used $1 / 25$ in our calculations. The range for $x_{F}$ changes between $1 / 25$ to $1 / 15$. Finally, the relic density of neutralinos at present time is defined as

$$
\Omega_{\chi} h^{2}=\frac{\rho\left(T_{0}\right)}{\rho_{c}},
$$

where $\rho\left(T_{0}\right)=m_{\chi} n_{\chi}\left(T_{0}\right)$ is the density of the neutralino, and $\rho_{c}=3 h^{2} M_{\mathrm{Pl}}^{2} / 8 \pi=8.098 \times 10^{-47} \mathrm{GeV}^{4}$ is the critical density of the universe. On the left-hand side of the equation, $h$ is the normalized Hubble expansion rate and its value today is 0.71 . Thus, from the central value of $\Omega_{\chi} h^{2}$, $\Omega_{\chi}$ is found to be about $22 \%$.

To obtain our final results, a three-dimensional integration needs to be carried out numerically. The three parts are an integration over the Mendelstam variable $t$ to compute $\sigma(s)$ for each subprocess in the kinematically allowed region, an integration over the center of mass energy squared $s$ from the threshold to practically infinity, and finally an integration over $x$ to compute the thermal averaging from the freeze-out temperature to today's $\left(x_{0}\right)$, which we approximate to zero. MATHEMATICA is used for the computation and the matrix element calculations have been carried out with FEYNCALC [31].

The Feynman diagrams contributing to the annihilation cross section are given in Fig. 5. We analyze here pure state contributions only, while any mixed case scenario can be calculated in a straightforward manner. Since our analysis concentrates on the non-MSSM scenarios, the MSSM contributions are not shown here. Depending of the center of mass energy available, the resonance problem in $s$-channel is handled using the Breit-Wigner prescription.

The mass of the $Z_{R}$ boson in diagram (a) is $M_{Z_{R}}=$ $\frac{g \cos \theta_{W}}{\sqrt{\cos 2 \theta_{W}}}\left(v_{\delta}^{2}+v_{\Delta}^{2}\right)^{1 / 2}$ [26]. In diagram (b) we sum over all left- and right-handed quarks and leptons in the final state. The sfermion $\tilde{f}$ has a (mass) ${ }^{2}$ given by, for $U$-type squarks and sneutrinos, 


$$
\mathcal{M}_{U_{k}}^{2}=\left(\begin{array}{cc}
M_{\mathrm{SUSY}}^{2}+M_{Z_{L}}^{2}\left(T_{u}^{3}-Q_{u} \sin ^{2} \theta_{W}\right) \cos 2 \beta & m_{u_{k}}(A-\mu \cot \beta) \\
m_{u_{k}}(A-\mu \cot \beta) & M_{\mathrm{SUSY}}^{2}+M_{Z_{L}}^{2} Q_{u} \sin ^{2} \theta_{W} \cos 2 \beta
\end{array}\right),
$$

and for $D$-type squarks and sleptons,

$$
\mathcal{M}_{D_{k}}^{2}=\left(\begin{array}{cc}
M_{\mathrm{SUSY}}^{2}+M_{Z_{L}}^{2}\left(T_{d}^{3}-Q_{d} \sin ^{2} \theta_{W}\right) \cos 2 \beta & m_{d_{k}}(A-\mu \tan \beta) \\
m_{d_{k}}(A-\mu \tan \beta) & M_{\mathrm{SUSY}}^{2}+M_{Z_{L}}^{2} Q_{d} \sin ^{2} \theta_{W} \cos 2 \beta
\end{array}\right) .
$$

Here $M_{\text {SUSY }}$ represents the universal scalar mass, $A$ and $\mu$ the trilinear and bilinear scalar parameters, respectively, $m_{u, d}$ are quark masses and the index $k$ labels generations. It is customary to restrict the supersymmetric parameter space by drawing contours in the $M_{1 / 2}-M_{\text {SUSY }}$ plane, where $M_{1 / 2}$ is the relevant gaugino or Higgsino mass parameter and $M_{\text {SUSY }}$ the relevant scalar mass. When the bino or right-wino are the LSP, we take their masses to be the significant gaugino mass. In diagram (c) the final state $h^{0}$ is the MSSM-like lightest Higgs boson, which we constrain to have a mass $M_{h^{0}}=115 \mathrm{GeV}$. In LRSUSY, its (mass) ${ }^{2}$ is given by the lowest eigenvalue of the matrix [26]:

$$
\mathcal{M}_{\phi_{22}, \phi_{11}}^{2}=\left(\begin{array}{cc}
m_{\phi_{1} \phi_{2}}^{2} \cot \beta-g^{2} v^{2} & -m_{\phi_{1} \phi_{2}}^{2} \\
-m_{\phi_{1} \phi_{2}}^{2} & m_{\phi_{1} \phi_{2}}^{2} \tan \beta+g^{2} v^{2}
\end{array}\right),
$$

where $v^{2}=v_{\delta}^{2}+v_{\Delta}^{2}+\kappa_{1}^{2}+\kappa_{2}^{2}$ and $m_{\phi_{1} \phi_{2}}$ is the Higgs mass parameter. Note that, while the cross sections for the decay of $\lambda_{V}, \lambda_{R}^{0}$ depend on $M_{\mathrm{SUSY}}$, the one for the $\tilde{\delta}_{R}^{0}$ Higgsino does not; we must find another relevant parameter for that case.

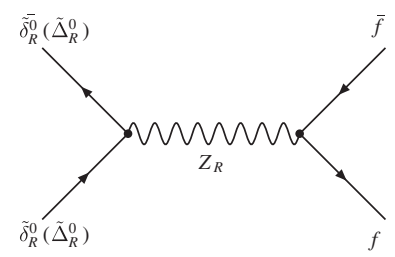

(a)

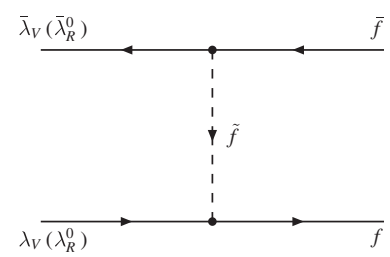

(b)

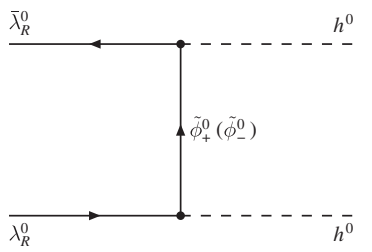

(c)

FIG. 5. The annihilation Feynman diagrams contributing $\tilde{\chi}_{1}^{0} \bar{\chi}_{1}^{0} \rightarrow \mathrm{X}_{\mathrm{SM}}$ in the LRSUSY model. The MSSM contributions and $u$-channel diagrams are not shown here. Contributions are given in the basis of pure states whose mixtures can be easily constructed. Here $f$ represent all fermions, and $\tilde{f}$ all sfermions, $h^{0}$ is the SM-like Higgs particle, and $\tilde{\phi}_{+}^{0}, \tilde{\phi}_{-}^{0}$ are the rotated Higgsinos fields defined in the previous section.

\section{NUMERICAL ANALYSIS}

Based on our classification of some possible mass scenarios in Sec. III, we analyze the first three mass scenarios with the lightest neutralino as a pure bino, right-wino, or Higgsino state. The feasibility of the last three scenarios, assuming mixed gaugino-Higgsino states, can be analyzed in the light of the pure state predictions. Throughout the numerical calculations, we have chosen the parameters of the model such that the $\Delta \rho$ bound, taken as $\Delta \rho \leq 0.002$ [28], is always satisfied.

Figure 6 shows the relic density $\Omega_{\chi} h^{2}$ of the neutralino as a pure bino state in the $M_{V}-M_{\text {SUSY }}$ plane for $\mu_{1}=$ $500 \mathrm{GeV}, A=200 \mathrm{GeV}$, and $\tan \beta=2$. There is no $s$-channel contribution and the $t$-channel fermion pair production $\lambda_{V} \bar{\lambda}_{V} \rightarrow \tilde{f} f \bar{f},(f=u, c, t, d, b, s \ldots)$ is the only

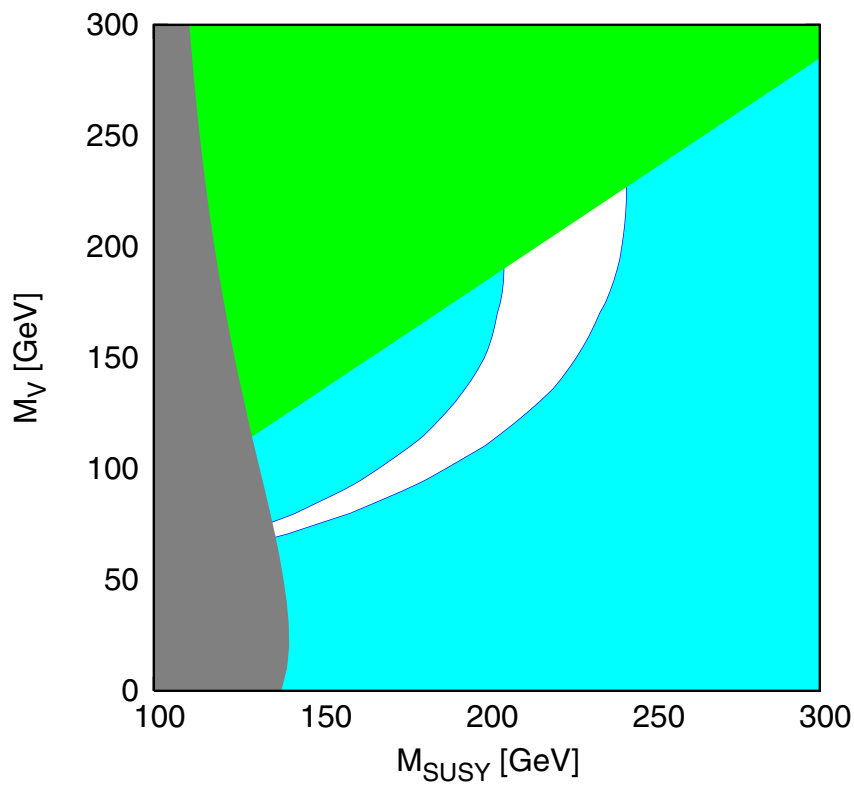

FIG. 6 (color online). The relic density of a pure bino state in the $M_{V}-M_{\text {SUSY }}$ plane for $\mu_{1}=500 \mathrm{GeV}, A=200 \mathrm{GeV}$, and $\tan \beta=2$, in the scenario in which the bino is the lightest neutralino. The upper triangular region in green is excluded by the lower bound requirement of the lightest neutralino mass from LEP2 and by the lower bound on the lightest sfermion mass constraint. The light blue region is excluded by WMAP measurements. The gray vertical strip on the left is excluded by $b \rightarrow$ $s \gamma$. The white strip agrees with the WMAP at the $2 \sigma$ level. The anomalous magnetic moment of the muon is satisfied within the entire region. 
channel available. ${ }^{6}$ Because of the $s$-wave suppression, the fermion pair production for heavy fermions is dominant, but we include all possible channels. Unlike the MSSM bino, the $B-L$ bino does not couple to $W^{+} W^{-}$pairs or to Higgs pairs. For the exchanged sfermions, a flavor conserving scenario with a common scale $M_{\text {SUSY }}$ is assumed. In the figure, the upper triangular region in green is excluded by the lower mass bound requirement on the lightest neutralino from LEP2 and by the lower bound of the lightest sfermion mass determined mainly by $M_{\text {SUSY }}$. (We calculated the masses of the sfermions and require that they are at least $15 \mathrm{GeV}$ higher than the mass of the bino to avoid coannihilation channels.) The light blue regions are excluded by the WMAP measurements. In addition, the gray vertical strip on the left is excluded by the branching ratio of $b \rightarrow s \gamma$ upper bound, for which the formulas are given in the Appendix. We assumed that $2.4 \times 10^{-4} \leq$ $B R(b \rightarrow s \gamma) \leq 4.1 \times 10^{-4}$ range is allowed. In numerical calculation, we used the following set of parameter values, consistent with pure bino scenario: $M_{L}=M_{R}=3 \mathrm{TeV}$, $m_{\tilde{g}}=400 \mathrm{GeV}, A=200 \mathrm{GeV}, \mu_{1}=500 \mathrm{GeV}, \mu_{2}=$ $4 \mathrm{TeV}, v_{\delta}=100 \mathrm{GeV}$, and $v_{\Delta}=1.5 \mathrm{TeV}{ }^{7}$ As seen from the figure the $B R(b \rightarrow s \gamma)$ constraint excludes the light $M_{\text {SUSY }}(\leq 150 \mathrm{GeV}$ ) region. We also take into account the anomalous magnetic moment of muon $a_{\mu}=$ $(g-2)_{\mu} / 2$, where the analytical formulas are given in the Appendix. The dominant chargino-sneutrino and neutralino-smuon loop contributions are included. In numerical computation, like $B R(b \rightarrow s \gamma)$, the same parameter set is assumed and the range for the deviation from the $\mathrm{SM},-0.53 \times 10^{-10} \leq \Delta a_{\mu} \leq 44.7 \times 10^{-10}$, at $95 \%$ C.L. is used based on $e^{+} e^{-}$data (see [32] and references therein). $\Delta a_{\mu}$ depends mainly on the chosen values of $A$, $\mu_{1}, \tan \beta$, and $M_{\mathrm{SUSY}}$. We observe that it remains in the allowed range throughout the entire $\left(M_{\mathrm{SUSY}}, M_{V}\right)$ interval considered, as long as $\mu_{1} \tan \beta-A \geq 0$ holds.

Consequently, the white strip is the only region satisfying the WMAP at the $2 \sigma$ level. A realization of such a scenario requires light sfermion masses in the range $\sim 150-240 \mathrm{GeV}$ with an acceptable LSP mass in the region $\sim 60-220 \mathrm{GeV}$. In the light blue region on the right, the relic density $\left.\Omega_{\chi} h^{2}\right|_{\chi=\lambda_{V}}$ becomes larger than experimentally allowed values, while in the left painted region it is smaller than experimental requirements. This scenario can only be considered feasible in frameworks where there is at least one light sfermion, like stop $\tilde{t}$, or stau $\tilde{\tau}$.

Next, in Fig. 7 we show the relic density $\Omega_{\chi} h^{2}$ of the neutralino as a pure right-wino state in the $M_{R}-M_{\mathrm{SUSY}}$ plane for $\mu_{1}=1 \mathrm{TeV}, A=200 \mathrm{GeV}$, and $\tan \beta=2$. The

\footnotetext{
${ }^{6} \mathrm{We}$ recall that the bino here is the fermionic partner of the $U(1)_{B-L}$ gauge boson and is different from the bino in the MSSM.

${ }^{7}$ We ensure at all times that the bino is not just the lightest neutralino, but a pure state.
}

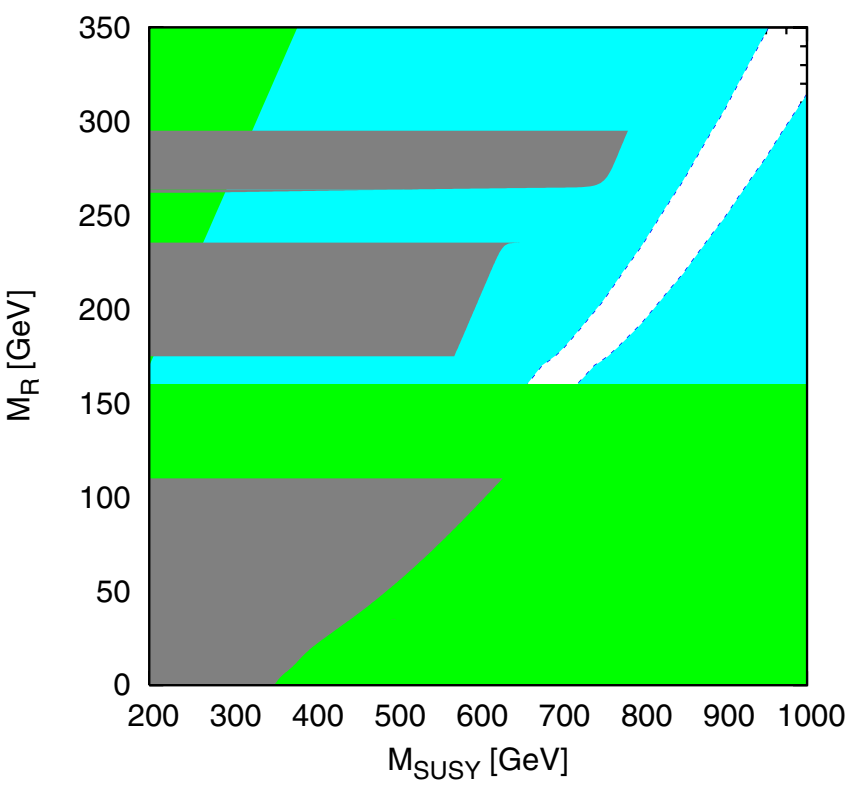

FIG. 7 (color online). The relic density of a pure right-wino state in the $M_{R}-M_{\text {SUSY }}$ plane for $\mu_{1}=1 \mathrm{TeV}, A=200 \mathrm{GeV}$, and $\tan \beta=2$ in the scenario in which the right-wino is the lightest neutralino. The left upper small triangular and the lower rectangular regions in green are excluded by the lower bound mass requirement on the lightest neutralino from LEP 2 and by the lower bound on the lightest sfermion mass. The light blue region is excluded by WMAP measurements. The gray region is excluded by $b \rightarrow s \gamma$. The white strip agrees with the WMAP at the $2 \sigma$ level. The anomalous magnetic moment of the muon is satisfied within the entire region.

right-wino as a neutralino is a completely new possibility peculiar to the LRSUSY model. There is no $s$-channel contribution and the possible annihilation contributions are $\quad \lambda_{R}^{0} \bar{\lambda}_{R}^{0} \rightarrow \tilde{f} f \bar{f}, \quad(f=u, c, t, d, b, s \ldots) \quad$ and $\lambda_{R}^{0} \bar{\lambda}_{R}^{0} \rightarrow \tilde{\phi}_{ \pm}^{0} h^{0} h^{0}$, when kinematically allowed. An important feature in this case is that there exists an additional condition - the LEP lower bound for the lightest chargino, which we take as $90 \mathrm{GeV}$. We also ensure that the lightest chargino remains always heavier than the lightest neutralino, at least $15 \mathrm{GeV}$ heavier, to avoid significant contributions from coannihilation channels. For the mixing in the chargino sector, we assume all parameters (except $M_{R}$ and $v_{\delta}$ ) large enough to obtain a pure right-wino state. Using $v_{\delta}=30 \mathrm{GeV}$, a $100 \mathrm{GeV}$ or larger mass is obtained for the right-wino, while fulfilling the bound on the mass of the lightest chargino. In order to obtain a neutralino lighter than the lightest chargino, $M_{R}$ should be at least $160 \mathrm{GeV}$ or larger; hence, the lower rectangular excluded region (in green) in Fig. 7. For illustrative purposes only, we have included, in Table I, the values of the masses of the LSP, as well as the masses of the second lightest neutralino, the lightest chargino, and the lightest sfermion, for all three scenarios investigated. As in the case of pure bino, the mass upper bound comes from sfermion sector. After implementing WMAP constraint, the only surviving region 
TABLE I. Representative values for the masses of the LSP $\left(\chi_{1}^{0}\right)$, the next-lightest neutralino $\left(\chi_{2}^{0}\right)$, lightest chargino $\left(\chi_{1}^{ \pm}\right)$, and the lightest sfermion $\left(\tilde{f}_{1}\right)$ for the three scenarios described in this section. The masses are given in GeV. The parameter sets are $\left(M_{L}, M_{R}, M_{V}, \mu_{1}, \mu_{2}, M_{\mathrm{SUSY}}, v_{\delta}\right)=(1,1,0.13,1,2,0.2,0.05) \mathrm{TeV}$ for Set (1), $\left(M_{L}, M_{R}, M_{V}, \mu_{1}, \mu_{2}, M_{\mathrm{SUSY}}, v_{\delta}\right)=(1,0.2,1,1,4,0.8,0.01) \mathrm{TeV} \quad$ for $\quad$ Set $\quad$ (2), and $\left(M_{L}, M_{R}, M_{V}, \mu_{1}, \mu_{2}, M_{\mathrm{SUSY}}, v_{\delta}\right)=(2,2,2,2,0.85, *, 0.1) \mathrm{TeV}$ for Set (3). For all three sets, $\tan \beta=2, A=0.2 \mathrm{TeV}$, and $v_{\Delta}=1.5 \mathrm{TeV}$ are used. “*” indicates that the entry depends on $M_{\text {SUSY }}$ which can be taken arbitrarily large.

\begin{tabular}{lccccccc}
\hline \hline Scenario & LSP & $M_{\chi_{1}^{0}}$ & $M_{\chi_{2}^{0}}$ & $M_{\chi_{1}^{ \pm}}$ & $M_{\tilde{f}_{1}}$ & $\Omega_{\chi} h^{2}$ & Parameter set \\
\hline 1 & bino & 130.6 & 472.2 & 500.0 & 176.2 & 0.106 & Set (1) \\
2 & $R$-wino & 177.1 & 924.5 & 196.4 & 713.4 & 0.121 & Set (2) \\
3 & $\tilde{\delta}_{R}^{0}$ Higgsino & 412.5 & 1426.5 & 713.4 & $*$ & 0.115 & Set (3) \\
\hline \hline
\end{tabular}

at the $2 \sigma$ level is shown in the white strip. This scenario satisfies the WMAP conditions for a relatively large $M_{\text {SUSY }}$ scale, $600 \mathrm{GeV}$ or larger. Note that the upper bound for the mass of the right-wino is closely related to the chosen values for the other gaugino masses, the vev's, and $\mu_{2}$, assumed heavy. For larger $M_{R}$ values, the lightest neutralino will not longer be a pure state. But one can see from Fig. 7 that it is not difficult to satisfy the WMAP result. Here too the constraints $b \rightarrow s \gamma$ and $(g-2)$ of the muon are taken into account with the parameter set, $M_{L}=M_{V}=$ $1.5 \mathrm{TeV}, m_{\tilde{g}}=400 \mathrm{GeV}, A=200 \mathrm{GeV}, \mu_{1}=500 \mathrm{GeV}$, $\mu_{2}=4 \mathrm{TeV}, v_{\delta}=100 \mathrm{GeV}$, and $v_{\Delta}=1.5 \mathrm{TeV}$. The $b \rightarrow s \gamma$ constraint for this case excludes mainly $M_{\mathrm{SUSY}} \leq$ $600 \mathrm{GeV}$ region but practically does not effect the WMAP allowed region. $(g-2)_{\mu}$ does not constrain the parameter space, as a light right-wino does not contribute signifi-

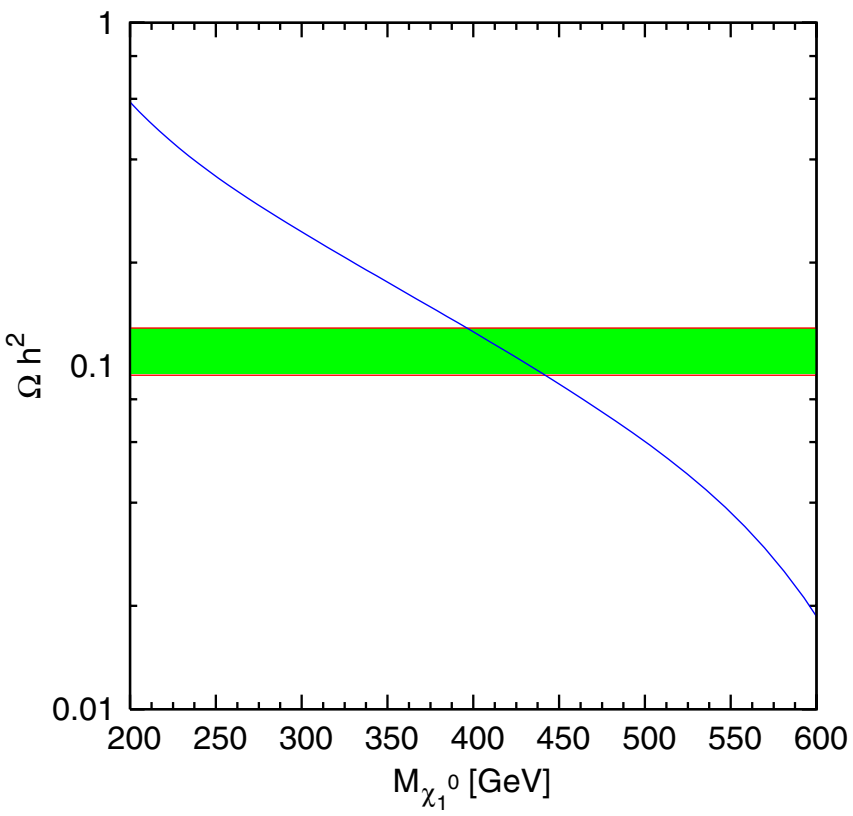

FIG. 8 (color online). The relic density of a pure Higgsino $\tilde{\delta}_{R}^{0}$ as a function of its mass for $v_{\delta}=100 \mathrm{GeV}, v_{\Delta}=1.5 \mathrm{TeV}$, in the scenario in which the Higgsino is the lightest neutralino. The green strip is the allowed region by the WMAP at the $2 \sigma$ level. cantly to the muon anomalous magnetic moment, and the other neutralinos are heavy.

In the third scenario we investigate the possibility that the $\tilde{\delta}_{R}^{0}$ is the lightest neutralino. This an interesting scenario, as $\tilde{\delta}_{R}^{0}$ is only introduced to cancel anomalies in the fermionic sector, and, because of its $B-L$ quantum number, its direct interactions with matter are forbidden. The relic density prediction for such scenario is shown in Fig. 8, as a function of the Higgsino mass $M_{\tilde{\delta}_{R}^{0}}$, for $v_{\delta}=$ $100 \mathrm{GeV}, v_{\Delta}=1.5 \mathrm{TeV}$. Only the $s$-channel contribution is available for the annihilation process $\tilde{\delta}_{R}^{0} \overline{\tilde{\delta}}_{R}^{0} \rightarrow Z_{R} f \bar{f}$, $(f=u, c, t, d, b, s \ldots)$. Note that, for this process, the cross section and thus the relic density is formally independent of $M_{\text {SUSY }}$, since $\tilde{\delta}_{R}^{0}$ cannot interact with sfermions. The $\Delta \rho$ bound does not allow values for $v_{\Delta}$ smaller than $1.5 \mathrm{TeV}$, once we assume a relatively small $v_{\delta}$. Then the mass of the exchange particle $Z_{R}$ becomes heavy, around $1.7 \mathrm{TeV}$, yielding suppressed cross sections. This is why we obtain a large relic density $\left.\Omega_{\chi} h^{2}\right|_{\chi=\delta_{R}^{0}}$, which is inversely proportional to the thermally averaged cross section. Only larger masses for the pure Higgsino lead to smaller relic density values. In that case, as well as having more phase space available, one is closer to the $Z_{R}$ resonance, which increases the annihilation cross section. So, as seen from the figure, the WMAP at the $2 \sigma$ level is satisfied if the mass of the Higgsino lies in 400-450 GeV narrow range. Neither $b \rightarrow s \gamma$ nor $(g-2)_{\mu}$ receive contributions from a $\tilde{\delta}_{R}^{0}$ Higgsino. Unfortunately the WMAP allowed region at $2 \sigma$ level restricts the LSP $\tilde{\delta}_{R}^{0}$ mass to a very small interval.

We will summarize our result in the next section.

\section{CONCLUSION}

In this study we have considered the neutralino sector of the LRSUSY model and concentrated on the lightest neutralino state as dark matter, motivated from the fact that WMAP result requires considerations of beyond MSSM models. Now, we would like to summarize our findings.

For the pure states in the LRSUSY model; if the masses of the supersymmetric partners (in both bosonic and fermionic sectors) are very small, the lightest neutralino is 
most likely to be a pure $B-L$ bino. This covers regions with $M_{\chi_{1}^{0}} \sim 60-220 \mathrm{GeV}$, and $M_{\mathrm{SUSY}} \sim 150-250 \mathrm{GeV}$. For intermediate to larger values for the masses of the supersymmetric partners, the lightest neutralino is most likely to be pure right-wino; this covers regions with $M_{\chi_{1}^{0}} \sim 150-350 \mathrm{GeV}$, and $M_{\mathrm{SUSY}} \sim 600-1000 \mathrm{GeV}$. Finally if the mass of the lightest neutralino is large, in the 400-450 GeV region, the state could be pure $\tilde{\delta}_{R}^{0}$-Higgsino. The mass region is severely restricted for pure Higgsino masses, but completely independent of $M_{\mathrm{SUSY}}$, as long as $M_{\mathrm{SUSY}}>500 \mathrm{GeV}$, so that none of the sfermions are lighter than the lightest neutralino.

The analysis above shows that the lightest neutralino as pure bino shares some features with the similar scenario in MSSM. Both are possible only if the scalars are light, especially for $M_{V} \sim 60 \mathrm{GeV}$. Outside this region, one obtains a relic density that is too large. This similarity is not unexpected: although the bino here is the fermionic partner of the $B-L$ gauge boson, and not the hypercharge gauge boson, the same dominant decay channels are open to both binos, so the cross sections are of the same order of magnitude.

The situation changes when we analyze the case in which the lightest neutralino is the right-wino. In MSSM, the case where the lightest neutralino is the left-wino requires a left-wino mass of $\sim 2.5 \mathrm{TeV}$ to satisfy dark matter limits. For our case, right-wino masses in the 150-350 GeV regions are in good agreement with the relic density constraints. The reason for this manifest difference is that the MSSM left-wino cross section is dominated by the decay into $W_{L}^{+} W_{L}^{-}$pairs, which is not available for the right-wino.

The $s$-channel is available only for the $\tilde{\delta}_{R}^{0}$-Higgsino, but this channel is suppressed by the large mass of the $Z_{R}$ boson propagator. The cross section is too small for light neutralinos, and the relic density is too large. It is less likely that the lightest neutralino would be the $\tilde{\delta}_{R}^{0}$-Higgsino, since only a very small mass range satisfies the dark matter constraints. In MSSM, the LSP is even less likely to be the (MSSM) Higgsino (see the recent study [33]). In LRSUSY, its annihilation cross section into $W_{L}$ and $Z_{L}$ gauge boson pairs is unsuppressed, and the relic density is too small, unless $\mu_{1}>1 \mathrm{TeV}$.

Our scenarios are very different from those present in the NMSSM or in other extensions of the MSSM, where a very light bino, or a singlino, or their mixture could be the LSP [12-14]. There the cross section is dominated by a light $C P$-odd Higgs boson or additional Higgs resonances, and these particles only couple to no SM particles except for the Higgs doublets.

\section{ACKNOWLEDGMENTS}

The work of D. D. was partially supported by Turkish Academy of Sciences through a GEBIP grant, and by the
Scientific and Technical Research Council of Turkey through Project No. 104T503. The work of M. F. and I. T. was funded in part by NSERC of Canada (SAP0105354). The authors thank Goran Senjanovič for useful email exchange. M. F. and I. T. would like to thank Geneviève Bélanger for useful comments. I. T. would also like to thank Marc Sher for helpful discussions.

\section{APPENDIX: CONSTRAINTS}

We include, for completeness, the constraints on the parameter space coming from the anomalous magnetic moment of the muon $\Delta a_{\mu}$ and the branching ratio for $b \rightarrow$ $s \gamma$. Constraints coming from $B_{s} \rightarrow \mu^{+} \mu^{-}$are more important than those coming from $b \rightarrow s \gamma$ only for $\tan \beta>$ 11 [34], which does not affect the calculation for our choice of parameters. For larger $\tan \beta$ values, we expect the values for the masses of the supersymmetric partners to shift to larger values.

In addition to $b \rightarrow s \gamma$ and $\Delta a_{\mu}$, we have included constraints form the LEP limits on supersymmetric masses; we have constrained the mass of the next-to-lightest supersymmetric particle to be at least $15 \mathrm{GeV}$ heavier than the lightest neutralino mass (to avoid coannihilations); and we have restricted the Higgs vev's so the $\rho$ parameter

$$
\begin{aligned}
\rho^{-1} & =\frac{M_{Z_{L}}^{2} \cos ^{2} \theta_{W}}{M_{W_{L}}^{2}} \\
& =1-\frac{1}{4} \frac{\cos ^{2} 2 \theta_{W}}{\cos ^{4} \theta_{W}} \frac{\kappa_{1}^{2}+\kappa_{2}^{2}}{v_{\Delta}^{2}+v_{\delta}^{2}}+\mathcal{O}\left[\left(\frac{\kappa_{1}^{2}+\kappa_{2}^{2}}{v_{\Delta}^{2}+v_{\delta}^{2}}\right)^{2}\right]
\end{aligned}
$$

is consistent with its experimental limits $\rho=0.9998_{-0.0010}^{+0.0025}$ at the $2 \sigma$ level [28].

$$
\text { 1. }(g-2)_{\mu}
$$

In addition to the relic density, another experimental result that can constrain the $M_{1 / 2}-M_{\text {SUSY }}$ parameter region is the BNL E821 measurement of the muon anomalous magnetic moment $a_{\mu} \equiv(g-2)_{\mu} / 2$ [35]:

$$
a_{\mu}(\exp )=11659208(6) \times 10^{-10},
$$

which indicates that the anomalous magnetic moment may need additional contributions beyond the SM to be consistent with the experimental values. There are however uncertainties in how big the deviation from SM really is. A $2.4 \sigma$ deviation is obtained if the SM prediction of hadronic contributions from $e^{+} e^{-}$data is used; if instead we use the data from indirect hadronic $\tau$ decays the disagreement with the muon anomalous magnetic moment is reduced to a $0.9 \sigma$ deviation. We will use, from the $95 \%$ C.L. range [32],

$$
-0.53 \times 10^{-10} \leq \Delta a_{\mu} \leq 44.7 \times 10^{-10} .
$$

The dominant contributions to $a_{\mu}$ in LRSUSY come from the chargino-muon sneutrino and neutralino-smuon loops. 
These are [36] $a_{\mu}=a_{\mu}^{\chi^{ \pm}}+a_{\mu}^{\chi^{0}}$, where

$$
a_{L \mu}^{\chi^{ \pm}}=\sum_{k=1}^{4} \frac{m_{\mu}}{16 \pi^{2}} M_{\chi_{k}^{ \pm}} g Y_{\mu} \operatorname{Re}\left[V_{k 1} U_{k 3}\right] \frac{F_{3}\left(x_{k \mu}\right)}{m_{\tilde{\nu}_{\mu}}^{2}}
$$

is the chargino contribution. The dominant neutralino contribution coming from neutralino-left slepton graphs is

$$
\begin{aligned}
a_{L \mu}^{\chi^{0}}= & \sum_{k=1}^{7} \frac{m_{\mu}}{16 \pi^{2}} M_{\chi_{k}^{0}} g\left[\sqrt { 2 } Y _ { \mu } \left(N_{k 1}\right.\right. \\
& \left.+\tan ^{2} \theta_{W} N_{k 3}\right) N_{5 k} \frac{F_{4}\left(y_{k \mu_{L}}\right)}{m_{\tilde{\mu}_{L}}^{2}}+g\left(N_{2 k}\right. \\
& \left.-2 \tan ^{2} \theta_{W} N_{k 3}\right)\left(N_{k 1}+\tan ^{2} \theta_{W} N_{k 3}\right) \\
& \left.\times \frac{m_{\mu}\left(A_{\mu}-\mu_{1} \tan \beta\right)}{m_{\tilde{\mu}_{R}}^{2}} \frac{F_{4}\left(y_{k \mu_{L}}\right)}{m_{\tilde{\mu}_{L}}^{2}}\right]
\end{aligned}
$$

and, from the neutralino-right slepton graphs,

$$
\begin{aligned}
a_{R \mu}^{\chi^{0}}= & \sum_{k=1}^{7} \frac{m_{\mu}}{16 \pi^{2}} M_{\chi_{k}^{0}} g\left[\frac { 1 } { \sqrt { 2 } } Y _ { \mu } \left(N_{k 2}\right.\right. \\
& \left.-2 \tan ^{2} \theta_{W} N_{k 3}\right) N_{k 5} \frac{F_{4}\left(y_{k \mu_{R}}\right)}{m_{\tilde{\mu}_{R}}^{2}}+g\left(N_{k 1}\right. \\
& \left.+\tan ^{2} \theta_{W} N_{k 3}\right)\left(N_{k 2}-2 \tan ^{2} \theta_{W} N_{k 3}\right) \\
& \left.\times \frac{m_{\mu}\left(A_{\mu}-\mu_{1} \tan \beta\right)}{m_{\tilde{\mu}_{L}}^{2}} \frac{F_{4}\left(y_{k \mu_{R}}\right)}{m_{\tilde{\mu}_{R}}^{2}}\right]
\end{aligned}
$$

with the loop functions $F_{3}(x), F_{4}(x)$ given in Eq. (A22) and where $x_{k \mu}=M_{\chi_{k}^{ \pm}}^{2} / m_{\tilde{\nu}_{\mu}}^{2}, y_{k \mu_{L, R}}=M_{\chi_{k}^{0}}^{2} / m_{\tilde{\mu}_{L, R}}^{2}$, and $U, V$ (and $N$ ) are matrices that diagonalize the chargino (and neutralino) mass matrices.

\section{2. $b \rightarrow s \gamma$}

The inclusive decay width for the process $b \rightarrow s \gamma$ is given by

$$
\Gamma(b \rightarrow s \gamma)=\frac{m_{b}^{5} G_{F}^{2} \alpha}{32 \pi^{4}}\left(\hat{M}_{\gamma L}^{2}+\hat{M}_{\gamma R}^{2}\right)
$$

where $\hat{M}$ means evolving down to the decay scale $\mu=m_{b}$. The branching ratio can be expressed as

$$
B R(b \rightarrow s \gamma)=\frac{\Gamma(b \rightarrow s \gamma)}{\Gamma_{S L}} B R_{S L},
$$

where the semileptonic branching ratio $B R_{S L}=B R(b \rightarrow$ $c e \bar{\nu})=(10.49 \pm 0.46) \%$ and

$$
\Gamma_{S L}=\frac{m_{b}^{5} G_{F}^{2}\left|\left(K_{\mathrm{CKM}}\right)_{c b}\right|^{2}}{192 \pi^{3}} g(z),
$$

where $\quad z=m_{c}^{2} / m_{b}^{2} \quad$ and $\quad g(z)=1-8 z+8 z^{3}-z^{4}-$ $12 z^{2} \log z$. The experimental measurement from CLEO can be expressed as [37]

$$
B R(b \rightarrow s \gamma)=\left(3.21 \pm 0.43 \pm 0.27_{-0.10}^{+0.18}\right) \times 10^{-4} \text {. }
$$

The SM contribution is

$$
\begin{aligned}
A_{\mathrm{SM}}= & \frac{\pi \alpha_{W}}{2 \sqrt{2} G_{F} M_{W}^{2}}\left(K_{\mathrm{CKM}}\right)_{t s}^{*}\left(K_{\mathrm{CKM}}\right)_{t b} 3 x_{t W}\left[Q_{u} F_{1}\left(x_{t W}\right)\right. \\
& \left.+F_{2}\left(x_{t W}\right)\right] .
\end{aligned}
$$

The matrix elements responsible for the $b \rightarrow s \gamma$ decay acquire the following contributions from the supersymmetric sector of the model [38]. For $b_{L}$ decay,

$$
M_{\gamma_{R}}=A_{\tilde{g}}^{R}+A_{\chi^{-}}^{R}+A_{\chi^{0}}^{R}
$$

with the gluino, chargino, and neutralino contributions given by

$$
\begin{aligned}
A_{\tilde{g}}^{R}= & -\frac{\sqrt{2} \pi \alpha_{s}}{G_{F}} Q_{d} C(R) \sum_{k=1}^{6} \frac{1}{m_{\tilde{d}_{k}}^{2}}\left\{\Gamma_{D L}^{k b} \Gamma_{D L}^{* k s} F_{2}\left(x_{\tilde{g} \tilde{d}_{k}}\right)\right. \\
& \left.-\frac{m_{\tilde{g}}}{m_{b}} \Gamma_{D R}^{k b} \Gamma_{D L}^{* k s} F_{4}\left(x_{\tilde{g} \tilde{d}_{k}}\right)\right\},
\end{aligned}
$$

$$
\begin{aligned}
A_{\chi^{-}}^{R}= & -\frac{\pi \alpha_{W}}{\sqrt{2} G_{F}} \sum_{j=1}^{5} \sum_{k=1}^{6} \frac{1}{m_{\tilde{u}_{k}}^{2}}\left\{\left(G_{U L}^{j k b}-H_{U R}^{j k b}\right)\left(G_{U L}^{* j k s}-H_{U R}^{* j k s}\right)\right. \\
& \times\left[F_{1}\left(x_{\chi_{j} \tilde{u}_{k}}\right)+Q_{u} F_{2}\left(x_{\chi_{j} \tilde{u}_{k}}\right)\right]+\frac{m_{\chi_{j}}}{m_{b}}\left(G_{U R}^{j k b}-H_{U L}^{j k b}\right) \\
& \left.\times\left(G_{U L}^{* j k s}-H_{U R}^{* j k s}\right)\left[F_{3}\left(x_{\chi_{j} \tilde{u}_{k}}\right)+Q_{u} F_{4}\left(x_{\chi_{j} \tilde{u}_{k}}\right)\right]\right\},
\end{aligned}
$$

$$
\begin{aligned}
A_{\chi^{0}}^{R}= & -\frac{\pi \alpha_{W}}{\sqrt{2} G_{F}} Q_{d} \sum_{j=1}^{9} \sum_{k=1}^{6} \frac{1}{m_{\tilde{d}_{k}}^{2}}\left\{\left(\sqrt{2} G_{0 D L}^{j k b}-H_{0 D R}^{j k b}\right)\right. \\
& \times\left(\sqrt{2} G_{0 D L}^{* j k s}-H_{0 D R}^{* j k s}\right) F_{2}\left(x_{\chi_{j}^{0} \tilde{d}_{k}}\right) \\
& +\frac{m_{\chi_{j}^{0}}}{m_{b}}\left(\sqrt{2} G_{0 D R}^{j k b}-H_{0 D L}^{j k b}\right) \\
& \left.\times\left(\sqrt{2} G_{0 D L}^{* j k s}-H_{0 D R}^{* j k s}\right) F_{4}\left(x_{\chi_{j}^{0} \tilde{d}_{k}}\right)\right\},
\end{aligned}
$$

and, for the decay of $b_{R}$,

$$
M_{\gamma_{L}}=A_{\tilde{g}}^{L}+A_{\chi^{-}}^{L}+A_{\chi^{0}}^{L}
$$

again, with the following gluino, chargino, and neutralino contributions:

$$
\begin{aligned}
A_{\tilde{g}}^{L}= & -\frac{\sqrt{2} \pi \alpha_{s}}{G_{F}} Q_{d} C(R) \sum_{k=1}^{6} \frac{1}{m_{\tilde{d}_{k}}^{2}}\left\{\Gamma_{D R}^{k b} \Gamma_{D R}^{* k s} F_{2}\left(x_{\tilde{g} \tilde{g}_{k}}\right)\right. \\
& \left.-\frac{m_{\tilde{g}}}{m_{b}} \Gamma_{D L}^{k b} \Gamma_{D R}^{* k s} F_{4}\left(x_{\tilde{g} \tilde{d}_{k}}\right)\right\}, \\
A_{\chi^{-}}^{L}= & -\frac{\pi \alpha_{W}}{\sqrt{2} G_{F}} \sum_{j=1}^{5} \sum_{k=1}^{6} \frac{1}{m_{\tilde{u}_{k}}^{2}}\left\{\left(G_{U R}^{j k b}-H_{U L}^{j k b}\right)\left(G_{U R}^{* j k s}-H_{U L}^{* j k s}\right)\right. \\
& \times\left[F_{1}\left(x_{\chi_{j} \tilde{u}_{k}}\right)+Q_{u} F_{2}\left(x_{\chi_{j} \tilde{u}_{k}}\right)\right] \\
& +\frac{m_{\chi_{j}}}{m_{b}}\left(G_{U L}^{j k b}-H_{U R}^{j k b}\right)\left(G_{U R}^{* j k s}-H_{U L}^{* j k s}\right) \\
& \left.\times\left[F_{3}\left(x_{\chi_{j} \tilde{u}_{k}}\right)+Q_{u} F_{4}\left(x_{\chi_{j} \tilde{u}_{k}}\right)\right]\right\},
\end{aligned}
$$




$$
\begin{aligned}
A_{\chi^{0}}^{L}= & -\frac{\pi \alpha_{W}}{\sqrt{2} G_{F}} Q_{d} \sum_{j=1}^{9} \sum_{k=1}^{6} \frac{1}{m_{\tilde{d}_{k}}^{2}}\left\{\left(\sqrt{2} G_{0 D R}^{j k b}-H_{0 D L}^{j k b}\right)\right. \\
& \times\left(\sqrt{2} G_{0 D R}^{* j k s}-H_{0 D L}^{* j k s}\right) F_{2}\left(x_{\chi_{j}^{0} \tilde{d}_{k}}\right) \\
& +\frac{m_{\chi_{j}^{0}}}{m_{b}}\left(\sqrt{2} G_{0 D L}^{j k b}-H_{0 D R}^{j k b}\right) \\
& \left.\times\left(\sqrt{2} G_{0 D R}^{* j k s}-H_{0 D L}^{* j k s}\right) F_{4}\left(x_{\chi_{j}^{0} \tilde{d}_{k}}\right)\right\},
\end{aligned}
$$

where the chargino-quark-squark mixing matrices $G$ and $H$ are defined as

$$
\begin{aligned}
G_{U L}^{j k i} & =V_{j 1}^{*}\left(\Gamma_{U L}\right)_{k i}, \quad G_{U R}^{j k i}=U_{j 2}\left(\Gamma_{U R}\right)_{k i}, \\
H_{U L}^{j k i} & =\frac{1}{\sqrt{2} m_{W}}\left(\frac{m_{u_{l}}}{\sin \beta}\left(K_{\mathrm{CKM}}\right)_{i l} U_{j 3}+\frac{m_{d_{l}}}{\cos \beta} \delta_{i l} U_{j 4}\right)\left(\Gamma_{U L}\right)_{k l}, \\
H_{U R}^{j k i} & =\frac{1}{\sqrt{2} m_{W}}\left(\frac{m_{u_{l}}}{\sin \beta}\left(K_{\mathrm{CKM}}\right)_{i l} V_{j 3}^{*}+\frac{m_{d_{l}}}{\cos \beta} \delta_{i l} V_{j 4}^{*}\right)\left(\Gamma_{U R}\right)_{k l},
\end{aligned}
$$

and where the neutralino-quark-squark mixing matrices $G_{0}$ and $H_{0}$ are defined as

$$
\begin{aligned}
G_{0 D L}^{j k i}= & \left\{-\left[\tan ^{2} \theta_{W}\left(T_{d}^{3}-Q_{d}-\frac{Q_{u}+Q_{d}}{2}\right)\right.\right. \\
& \left.+\frac{Q_{u}+Q_{d}}{2}\right] N_{j 1}^{*}-\frac{\sqrt{\cos 2 \theta_{W}}}{\cos \theta_{W}} \tan \theta_{W}\left(T_{d}^{3}-Q_{d}\right. \\
& \left.\left.-\frac{Q_{u}+Q_{d}}{2}\right) N_{j 2}^{*}+T_{d}^{3} N_{j 3}^{*}\right\}\left(\Gamma_{D L}\right)_{k i}, \\
G_{0 D R}^{j k i}= & \left\{-\frac{\cos 2 \theta_{W}}{\cos ^{2} \theta_{W}}\left(T_{d}^{3}-2 Q_{d} \sin ^{2} \theta_{W}\right) N_{j 1}\right. \\
& +\sqrt{\cos 2 \theta_{W}} \sin \theta_{W}\left[Q_{d}+\frac{1}{\cos ^{2} \theta_{W}}\right. \\
& \left.\left.\times\left(T_{d}^{3}-2 Q_{d} \sin ^{2} \theta_{W}\right)\right] N_{j 2}+2 \sin ^{2} \theta_{W} Q_{d} N_{j 3}\right\}\left(\Gamma_{D R}\right)_{k i}, \\
H_{0 D L}^{j k i}= & \frac{1}{\sqrt{2} m_{W}}\left(\frac{m_{u_{l}}}{\sin \beta} N_{j 4}-\frac{m_{d_{l}}}{\cos \beta} N_{j 5}\right)\left(\Gamma_{D L}\right)_{k i}, \\
H_{0 D R}^{j k i}= & \frac{1}{\sqrt{2} m_{W}}\left(\frac{m_{u_{l}}}{\sin \beta} N_{j 4}^{*}+\frac{m_{d_{l}}}{\cos \beta} N_{j 5}^{*}\right)\left(\Gamma_{D R}\right)_{k i}, \quad \text { (A21) }
\end{aligned}
$$

and where the matrices $\Gamma_{U, D}$ diagonalize the squark mass matrices in the up and down sectors. The functions appearing in the expressions above are

$$
\begin{aligned}
& F_{1}(x)=\frac{1}{12(x-1)^{4}}\left(x^{3}-6 x^{2}+3 x+2+6 x \log x\right), \\
& F_{2}(x)=\frac{1}{12(x-1)^{4}}\left(2 x^{3}+3 x^{2}-6 x+1-6 x^{2} \log x\right), \\
& F_{3}(x)=\frac{1}{2(x-1)^{3}}\left(x^{2}-4 x+3+2 \log x\right), \\
& F_{4}(x)=\frac{1}{2(x-1)^{3}}\left(x^{2}-1-2 x \log x\right) .
\end{aligned}
$$

The convention $x_{a b}=m_{a}^{2} / m_{b}^{2}$ is used. $C(R)=4 / 3$ is the quadratic Casimir operator of the fundamental representation of $S U(3)_{C}$.

In order to compare the results obtained with experimental branching ratios, QCD corrections must be taken into account. We assume the renormalization group evolution pattern. There is no mixing between left- and righthanded contributions:

$$
\begin{aligned}
A^{\gamma}\left(m_{b}\right)= & \eta^{-16 / 23}\left\{A^{\gamma}\left(M_{W}\right)+A_{0}^{\gamma}\left[\frac{116}{135}\left(\eta^{10 / 23}-1\right)\right.\right. \\
& \left.\left.+\frac{58}{189}\left(\eta^{28 / 23}-1\right)\right]\right\},
\end{aligned}
$$

where $\quad \eta=\alpha_{s}\left(m_{b}\right) / \alpha_{s}\left(M_{W}\right) \quad$ and $\quad A_{0}^{\gamma}=\frac{\pi \alpha_{W}}{2 \sqrt{2} G_{F} M_{W}^{2}} \times$ $\left(K_{\mathrm{CKM}}\right)_{t s}^{*}\left(K_{\mathrm{CKM}}\right)_{t b}$. We choose the renormalization scale to be $\mu=m_{b}=4.2 \mathrm{GeV}$.
[1] H. V. Peiris et al., Astrophys. J. Suppl. Ser. 148, 213 (2003); M. R. Nolta et al., Astrophys. J. 608, 10 (2004).

[2] H. Goldberg, Phys. Rev. Lett. 50, 1419 (1983); J. R. Ellis, J. S. Hagelin, D. V. Nanopoulos, K. A. Olive, and M. Srednicki, Nucl. Phys. B238, 453 (1984).

[3] K. A. Olive, hep-ph/0412054; C. Boehm, A. Djouadi, and M. Drees, Phys. Rev. D 62, 035012 (2000); J. R. Ellis, K. A. Olive, Y. Santoso, and V. C. Spanos, Phys. Lett. B 565, 176 (2003).

[4] N. Arkani-Hamed, A. Delgado, and G. F. Giudice, Nucl. Phys. B741, 108 (2006).

[5] K. Griest and D. Seckel, Phys. Rev. D 43, 3191 (1991).
[6] P. Gondolo, J. Edsjo, P. Ullio, L. Bergstrom, M. Schelke, and E. A. Baltz, J. Cosmol. Astropart. Phys. 07 (2004) 008.

[7] G. Belanger, F. Boudjema, A. Pukhov, and A. Semenov, Comput. Phys. Commun. 174, 577 (2006); Comput. Phys. Commun. 149, 103 (2002).

[8] M.E. Gomez, G. Lazarides, and C. Pallis, Phys. Lett. B 487, 313 (2000).

[9] U. Chattopadhyay, A. Corsetti, and P. Nath, Phys. Rev. D 68, 035005 (2003); R. Arnowitt, B. Dutta, B. Hu, and Y. Santoso, Phys. Lett. B 505, 177 (2001).

[10] V. D. Barger and C. Kao, Phys. Rep. 307, 207 (1998).

[11] H. Baer, T. Krupovnickas, A. Mustafayev, E. K. Park, S. 
Profumo, and X. Tata, hep-ph/0511034; H. Baer, A. Mustafayev, S. Profumo, A. Belyaev, and X. Tata, J. High Energy Phys. 07 (2005) 065.

[12] G. Belanger, F. Boudjema, C. Hugonie, A. Pukhov, and A. Semenov, J. Cosmol. Astropart. Phys. 09 (2005) 001; V. Barger, P. Langacker, and H. S. Lee, AIP Conf. Proc. 805, 306 (2005); V. Barger, C. Kao, P. Langacker, and H. S. Lee, Phys. Lett. B 600, 104 (2004); J. F. Gunion, D. Hooper, and B. McElrath, Phys. Rev. D 73, 015011 (2006).

[13] B. de Carlos and J. R. Espinosa, Phys. Lett. B 407, 12 (1997).

[14] V. Barger, P. Langacker, and H. S. Lee, Phys. Lett. B 630, 85 (2005).

[15] C.S. Aulakh, B. Bajc, A. Melfo, A. Rasin, and G. Senjanovic, Phys. Lett. B 460, 325 (1999); C. S. Aulakh, A. Melfo, A. Rasin, and G. Senjanovic, Phys. Lett. B 459, 557 (1999).

[16] K. Huitu and J. Maalampi, Phys. Lett. B 344, 217 (1995).

[17] M. Frank, M. Sher, and I. Turan, Phys. Rev. D 71, 113002 (2005); M. Frank, I. Turan, and M. Sher, Phys. Rev. D 71, 113001 (2005).

[18] B. Dutta, Y. Mimura, and R. N. Mohapatra, Phys. Rev. D 72, 075009 (2005).

[19] R. M. Francis, M. Frank, and C. S. Kalman, Phys. Rev. D 43, 2369 (1991).

[20] C.S. Aulakh, A. Melfo, and G. Senjanovic, Phys. Rev. D 57, 4174 (1998).

[21] Z. Chacko and R. N. Mohapatra, Phys. Rev. D 58, 015003 (1998); B. Dutta and R. N. Mohapatra, Phys. Rev. D 59, 015018 (1999).

[22] R. N. Mohapatra and G. Senjanovic, Phys. Rev. D 23, 165
(1981).

[23] C. S. Aulakh, K. Benakli, and G. Senjanovic, Phys. Rev. Lett. 79, 2188 (1997).

[24] M. Frank, Phys. Lett. B 540, 269 (2002).

[25] C.S. Aulakh, A. Melfo, A. Rasin, and G. Senjanovic, Phys. Rev. D 58, 115007 (1998).

[26] M. Frank and P. Pnevmonidis, Phys. Rev. D 67, 015010 (2003).

[27] M. Frank and I. Turan, Phys. Rev. D 72, 035008 (2005).

[28] S. Eidelman et al. (Particle Data Group), Phys. Lett. B 592, 1 (2004).

[29] P. Gondolo and G. Gelmini, Nucl. Phys. B360, 145 (1991).

[30] H. Baer, C. Balazs, A. Belyaev, and J. O'Farrill, J. Cosmol. Astropart. Phys. 09 (2003) 007; H. Baer, C. Balazs, and A. Belyaev, J. High Energy Phys. 03 (2002) 042.

[31] R. Mertig and J. Kublbeck, prepared for International Workshop on Software Engineering, Artificial Intelligence and Expert Systems for High-energy and Nuclear Physics, Lyon, France, 1990; J. Kublbeck, H. Eck, and R. Mertig, Nucl. Phys. A, Proc. Suppl. 29, 204 (1992).

[32] G. Lazarides, hep-ph/0601016.

[33] U. Chattopadhyay, D. Choudhury, M. Drees, P. Konar, and D. P. Roy, Phys. Lett. B 632, 114 (2006).

[34] M. Frank and S. Nie, Phys. Rev. D 66, 055001 (2002).

[35] G. W. Bennett et al. (Muon g-2 Collaboration), Phys. Rev. Lett. 92, 161802 (2004).

[36] M. Frank, Phys. Rev. D 59, 013003 (1999).

[37] S. Chen et al. (CLEO Collaboration), Phys. Rev. Lett. 87, 251807 (2001).

[38] M. Frank and S. Nie, Phys. Rev. D 65, 114006 (2002). 